\title{
Solidification of a binary alloy of variable viscosity from a vertical boundary
}

\author{
By RICHARD A. JARVIS $\dagger$ AND HERBERT E. HUPPERT $\ddagger$ \\ Institute of Theoretical Geophysics, Department of Applied Mathematics and Theoretical Physics, \\ University of Cambridge, Silver Street, Cambridge CB3 9EW, UK
}

(Received 17 October 1994 and in revised form 24 May 1995)

We analyse the complete solidification from a side boundary of a finite volume of a binary alloy. Particular emphasis is placed upon the compositional stratification produced in the solid, the structure of which is determined by the competition between the rates of solidification and of laminar box filling by the fractionated fluid released at the solid/liquid interface. It is demonstrated by scaling arguments that numerical calculations performed at relatively low values of the Rayleigh and Lewis numbers may be used to describe equally well laboratory experiments previously performed at moderate Rayleigh and Lewis numbers and the high-Rayleigh-number, high-Lewisnumber convective regime expected during the solidification of a large magmatic body, provided that the balance between solidification and laminar box filling is maintained. This balance can be represented by a single dimensionless group of parameters. The boundary-layer analysis is extended to fluids whose viscosity is strongly dependent upon temperature and composition, and an effective viscosity is derived which may be used to describe both the magnitude and pattern of compositional stratification in the solid.

\section{Introduction}

When a multicomponent melt solidifies, the composition of the solid alloy formed is generally different from the composition of the liquid from which it originates. Consequently, the fluid in the vicinity of the solidification front is depleted in those components which are preferentially incorporated into the solid phase, and enriched in those components which are rejected. The density of the liquid is generally dependent upon its composition, and thus solidification is often associated with density differences and hence convection in the fluid (Chen \& Turner 1980; Turner \& Gustafson 1981; Huppert 1990). The convective transport of heat and chemical species in turn alters the solidification process itself.

Here we investigate this interaction between solidification and convection for a binary fluid that is cooled from a side boundary. In particular, we focus on how the redistribution by convection of the two chemical components of the fluid can lead to spatial variations in the composition of the solid produced. This is of special interest to metallurgists, crystal growers and geologists, albeit for differing reasons. Metallurgists generally wish to suppress variations in the composition of the

$\dagger$ Present address: Laboratoire de Dynamique des Systèmes Géologiques, Institut de Physique du Globe, 4 place Jussieu, 75252 Paris Cedex 05, France.

$\ddagger$ Author to whom correspondence should be addressed. 
solid because impurities in a metal casting can significantly weaken the solid alloy. Similarly, crystal growers seek a homogeneous product.

Geologists, conversely, wish to interpret existing rock features to understand the physical processes which led to their formation. Igneous intrusions are formed by the crystallisation of magma within the crust and compositional stratification has been observed in many intrusive igneous bodies, for example the Skaergaard intrusion in east Greenland (McBirney \& Noyes 1979). Igneous layering, as this compositional stratification is commonly termed, can occur on a wide variety of spatial scales, although the model presented here is only able to address variations on the largest scale, which is comparable to that of the intrusion itself. A related geological problem is to determine the rate at which chemically fractionated magma is transported convectively from the vicinity of the solidification front into the interior of the magma body. Numerous studies of erupted materials point to the accumulation of relatively light, silica-rich, magma at the roof of evolving magma chambers (see, for example, Hildreth 1981).

The convection associated with solidification at a side boundary has in the past been investigated using three different approaches. The first group of studies is theoretical, and has concentrated upon the structure of the boundary-layer flow adjacent to the advancing solidification front. The basic similarity solution for natural convection at a fixed vertical plate (Ostrach 1953; Kuiken 1968) has been extended to fluids of variable viscosity (Spera, Yuen \& Kirschvink 1982) and to cases in which convection is driven both by temperature differences and by compositional differences created at the solidification front (Gebhart \& Pera 1982; Nilson \& Baer 1982; Nilson 1985; Nilson, McBirney \& Baker 1985). All these studies assume a semi-infinite fluid with a fixed far-field composition and temperature. An extended form of the similarity solution for constant-viscosity thermal convection which applies when the far-field fluid is stratified was derived by Worster \& Leitch (1985), who used their solution to describe the box-filling process which occurs when fractionated fluid detrains from the boundary layer and intrudes into the interior of a box of finite size.

The second approach taken has been to perform analogue laboratory experiments with aqueous salt solutions. Leitch $(1985,1987)$ and Huppert et al. (1987) crystallized aqueous sodium carbonate solutions from one side of a small tank, with a height of approximately $20 \mathrm{~cm}$. Their experiments demonstrated that the compositional stratification in the solid is strongly influenced by the thermal and compositional convection in the fluid. In particular, the overall pattern of composition in the solid can be explained by the rate at which laminar box filling, as described by Worster \& Leitch (1985), proceeds. However, the experiments were performed in a fluid with constant viscosity, which limits their immediate application to solidification in magma chambers.

Finally, there have been a large number of numerical calculations of solidifying alloys, primarily inspired by metallurgical applications, and of which we shall review briefly only a few. The simplest models involve pure melts (Ramachandran, Gupta \& Jaluria 1981; Ho \& Viskanta 1984), and demonstrate how the shape of the solidification front is altered by the convective transport of heat within the chamber. These models were extended to binary melts by Thompson \& Szekely $(1987,1988)$, who successfully simulated their laboratory experiments performed with aqueous sodium carbonate solutions. However, the cooling regime studied by Thompson \& Szekely $(1987,1988)$ did not lead to a compositionally variegated solid and their investigations focused upon the formation of double-diffusive layers when the chamber was heated at the opposite sidewall during solidification. More complicated 
models for the solidification of binary fluids, that seek to account for the presence of a two-phase mushy zone, have been formulated more recently, following the general description provided by Bennon \& Incropera (1987a).

A shortcoming common to all these numerical models is their inability to treat the large-Rayleigh-number, large-Lewis-number regime $\left(R a_{C} \sim 10^{15}-10^{18}, L e \sim 10^{3}\right)$ which is thought to be typical of solidifying magma chambers. This is generally blamed upon the limitations of present-day computers (a perennial complaint). Similarly, convection at such high Rayleigh and Lewis numbers cannot readily be achieved on the laboratory scale. Clearly a different approach is required if we are to address the geological regime. This paper is an attempt to bridge the gap by appealing to boundary-layer theory to provide scaling laws which enable us to simulate conditions in magma chambers with numerical calculations performed at much lower Rayleigh numbers. These scaling laws describe both the compositional stratification in the solid produced and the rate of fractionation, or box filling, in the fluid. After testing our scaling laws against the laboratory experiments discussed above, we are then able to extend our analysis to fluids of variable viscosity, which enables the constant-viscosity laboratory experiments to be more widely applied to geological situations.

The plan of the paper is as follows. First, in $\$ 2$, we outline the basic principles of the solidification of a binary alloy and describe the 'flat interface' approximation which enables us to calculate the macrosegregation in the solidified alloy. In $\S 3$, we then describe the governing equations and boundary conditions applicable to solidification of an alloy contained in a finite two-dimensional box, restricting compositional and thermal convection to be laminar. After a brief discussion of the expected structure of the double-diffusive boundary-layer flow adjacent to the solid/liquid interface, we perform a scaling analysis in $\S 4$ for fluids of constant viscosity to demonstrate the fundamental balances that determine the pattern and magnitude of compositional stratification in the final solid product, starting from the laminar box-filling theory of Worster \& Leitch (1985). In $\S 5$ we test our theory by performing low-Rayleigh-number numerical simulations of the laboratory experiments described by Leitch $(1985,1987)$ and by Huppert et al. (1987). With an application to magmatic systems in mind, we then treat fluids of variable viscosity in $\S 6$ by studying the structure of the inner compositional boundary layer. We derive, and test, an effective viscosity which can describe both the compositional stratification in the solid and the total upward flux of fractionated fluid to the roof of the chamber. Finally, in $\$ 7$, we summarize our main results and discuss their potential implications.

\section{Solidification of a binary alloy}

We restrict attention to a binary eutectic alloy, for which a schematic equilibrium phase diagram is presented in figure 1. The composition of the alloy is defined by a continuous scale between its two end-members $A$ and $B$. The solidification behaviour of the alloy depends upon which side of the eutectic composition, $C_{E}$, the fluid composition, $C$, lies, and hence the phase diagram has two branches, which correspond to alloys of subeutectic composition $\left(C_{A}<C<C_{E}\right)$ and supereutectic composition $\left(C_{E}<C<C_{B}\right)$ respectively. Fluid of eutectic composition $\left(C=C_{E}\right)$ behaves as a pure melt, crystallizing to form solid of eutectic composition.

Without loss of generality, we shall assume that end-member $B$ is the heavier component, so that compositionally heavier fluid is released at the solid/liquid interface when a subeutectic alloy solidifies, and compositionally light fluid is released when a supereutectic alloy solidifies. 


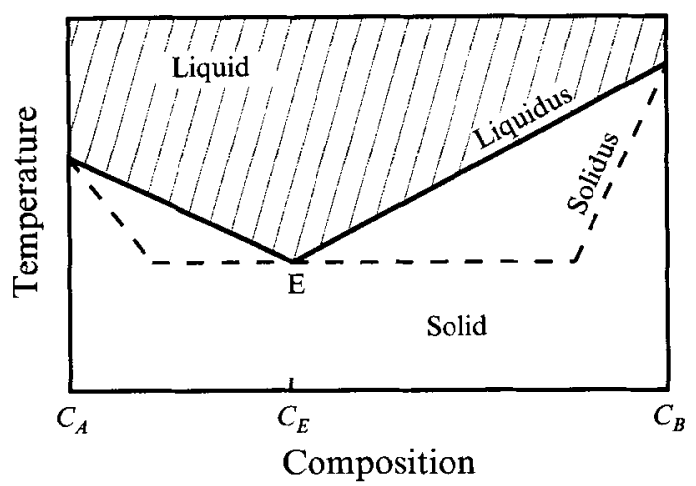

FIGURE 1. Schematic equilibrium phase diagram for a binary eutectic alloy. The composition of the alloy is defined on a continuous scale from pure $A$ to pure $B$, and the two branches of the liquidus curve (solid lines) mark the temperature above which the alloy is totally liquid at thermodynamic equilibrium. The composition of the solid formed at a given temperature is given by the solidus curve (dashed lines). At the eutectic point, $\mathrm{E}$, the alloy has eutectic composition $C_{E}$. The grey are lines of constant fluid density, and emphasize the greater effect upon density of compositional variations over thermal variations.

If solidification is occurring under conditions of thermodynamic equilibrium, the temperature and composition of the fluid adjacent to the solidification front are constrained to lie on the appropriate branch of the liquidus curve. Given the interfacial temperature, the composition of the solid produced is then determined by the corresponding solidus curve. For $C \neq C_{E}$, the preferential solidification of one or the other end-member results in a gradual evolution of the mean composition of the residual melt towards the eutectic composition as crystallization proceeds. Complete solidification of a binary eutectic alloy can only occur if it is cooled below its eutectic temperature.

To maintain local thermodynamic equilibrium in the fluid ahead of the solidification front, an advancing planar solid-liquid interface may become unstable (Mullins \& Sekerka 1964), forming a two-phase mushy zone in which dendrites of $B$ are bathed in interstitial melt which lies on the liquidus (see the review by Worster 1992). Behind the advancing mush front there is then a second, eutectic solidification front at which the interstitial melt has eutectic composition and crystallizes to form eutectic solid between the dendrites of $B$. Although thermodynamic equilibrium has been maintained throughout, the bulk composition of the final solid is neither end-member, nor eutectic.

The simplest mush model is that of Worster (1986), in which local equilibrium is maintained throughout the mush and the liquid just ahead of the mush is at or above its local liquidus temperature. In practice, however, some constitutional undercooling is required to drive solidification (Langer 1980), and the larger this undercooling, the thinner will be the mush layer (Kerr et al. 1990a).

In this paper, we adopt the limiting 'flat interface' approximation of Woods \& Huppert (1989). Mathematically, the interface is treated as locally flat with a clean interface between solid and liquid. The mush layer is formally assumed to have infinitesimal thickness, so that the interfacial liquid has the eutectic temperature and composition associated with the solid-mush interface. However, the bulk composition of the solid formed need not be eutectic because of the presence of the thin mush, and is instead controlled by mass transfer within the melt. 


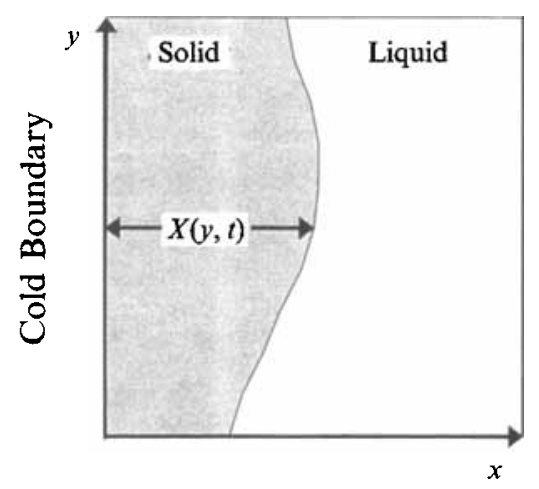

FIGURE 2. Schematic diagram of model fluid chamber. Solid grows from the cold boundary, $x=0$, and at time $t$ and height $y$ the solid has thickness $X(y, t)$. In general, the solidification front is not vertical because of the transport of heat by both thermal and compositional convection in the fluid.

Our reasons for choosing this approximation are twofold. First, we wish to draw comparisons with existing laboratory studies, for which the best documented cases are those conducted with aqueous sodium carbonate solutions, for which the mushy layer is usually very thin (Leitch 1985; Woods \& Huppert 1989). Second, this is a first attempt at obtaining quantitative agreement between experiment and theory, with a view to obtaining a more robust prediction for natural systems, and the flat interface is fluid-dynamically and mathematically simpler to treat. Ultimately, however, the appropriateness of the flat-interface approximation depends upon the system of interest. Woods \& Huppert (1989) obtain good agreement between measurements and predictions of solid composition using the flat-interface approximation for suband supereutectic sodium carbonate solutions, but the approximation would not be expected to work nearly so well for supereutectic aqueous ammonium chloride, which usually forms a thick dendritic mush (Huppert 1990). The flat interface is also a common approximation for metallurgical applications (Elliott 1977), although its applicability to magmatic crystallization is still to be tested.

\section{Convection driven by solidification at a vertical boundary}

\subsection{Mathematical model}

We consider a two-dimensional, rectangular box filled with a binary alloy which is initially liquid and uniform in temperature and composition (figure 2). At time $t=0$, the temperature of one vertical boundary $(x=0)$ is instantaneously dropped and maintained below the eutectic temperature of the melt, so that solidification of the alloy immediately commences at the cold boundary. All other boundaries remain insulated. We assume that the advancing interface between the solid and the liquid phases remains locally planar, and does not become morphologically unstable. Hence we may describe the solid/melt interface at time $t$ by the single-valued function $x=X(y, t)$, where $y$ is the vertical coordinate. We also assume that thermal and physical properties, such as heat capacity, are constant within each phase, with the notable exception of the fluid viscosity.

We assume that the resulting convection is laminar. For a fluid with local temperature $T_{l}$, composition $C_{l}$ and velocity $\boldsymbol{u}$, the Navier-Stokes equations under the 
Boussinesq approximation are

$$
\begin{gathered}
\rho_{l}\left(\frac{\partial \boldsymbol{u}}{\partial t}+\boldsymbol{u} \cdot \nabla \boldsymbol{u}\right)=-\nabla p+2 \nabla \cdot(\mu \mathbf{e})-\rho_{l} \boldsymbol{g}\left[\alpha\left(T_{l}-T_{0}\right)-\beta\left(C_{l}-C_{0}\right)\right] \\
\rho_{l} c_{p l}\left(\frac{\partial T_{l}}{\partial t}+\boldsymbol{u} \cdot \nabla T_{l}\right)=k_{l} \nabla^{2} T_{l}
\end{gathered}
$$

and

$$
\frac{\partial C_{l}}{\partial t}+\boldsymbol{u} \cdot \nabla C_{l}=\kappa_{C} \nabla^{2} C_{l}
$$

where $g$ is the acceleration due to gravity, $\kappa_{C}$ is the compositional diffusivity, and the fluid has dynamic viscosity $\mu$, density $\rho_{l}$, thermal capacity $c_{p l}$, thermal conductivity $k_{l}$, thermal coefficient of expansion $\alpha$ and compositional coefficient of expansion $\beta$. We have assumed that the fluid density varies linearly with composition and temperature. The local fluid pressure is $p$ and the rate-of-strain tensor $\mathbf{e}$ is defined by

$$
e_{i j}=\frac{1}{2}\left(\frac{\partial u_{i}}{\partial x_{j}}+\frac{\partial u_{j}}{\partial x_{j}}\right)
$$

We further assume that the fluid is incompressible, and so

$$
\nabla \cdot \boldsymbol{u}=0
$$

The temperature in the solid, $T_{s}$, is given by the diffusion equation

$$
\rho_{s} c_{p s} \frac{\partial T_{s}}{\partial t}=k_{s} \nabla^{2} T_{s}
$$

in which $\rho_{s}, c_{p s}$ and $k_{s}$ are the density, thermal capacity and thermal conductivity of the solid phase. We neglect compositional diffusion within the solid phase. Although we allow for differing densities of the solid and liquid phases $\left(\rho_{s} \neq \rho_{l}\right)$ in determining heat capacities per unit volume, we do not explore the implications of the contraction (or expansion) of the alloy upon solidification.

Initially, the fluid has uniform temperature $T_{l}=T_{0}$, composition $C_{l}=C_{0}$ and is motionless $(\boldsymbol{u}=0)$. At the cold boundary $x=0$, the solid temperature $T_{s}=T_{C}$, and across the remaining boundaries $(x=L, y=0$ and $y=H)$ there is no transfer of energy or mass $\left(\partial T_{l} / \partial n=\partial T_{s} / \partial n=\partial C_{l} / \partial n=0\right)$. We may also consider the region $0<x<L$ as one half of a fluid body cooled equally from both sides, since changing the boundary condition at $x=L$ from no slip to free slip has little noticeable effect on the following results. At the solid/liquid interface $x=X(y, t)$, and at the fixed boundaries $x=L, y=0$ and $y=H$, we apply the no-normal-flow and no slip conditions $\boldsymbol{u}=0$. Note that, although the solidification front is moving, there is no normal flow induced there. This is because fluid elements adjacent to the advancing solid/liquid interface are incorporated into the solid phase and we are discounting expansion (or contraction).

The solid composition is determined by the rate at which composition may be transported diffusively to and from the solidification front, and is given by the mass balance

$$
\left(C_{s}-C_{E}\right) \dot{X}=\kappa_{C} \frac{\partial C}{\partial n}
$$

where $\dot{X}$ is the rate of advance of the solidification front $x=X(y, t)$ and $\partial / \partial n$ is the normal derivative taken into the fluid. Compositional variegation in the solid can occur because of variations either in the solid growth rate, or in the diffusive 
flux of composition from the fluid. The temperatures of the solid and liquid and the composition of the liquid at the solid/liquid interface are

$$
T_{l}=T_{s}=T_{E} \quad \text { and } \quad C=C_{E} \quad \text { at } x=X(y, t) .
$$

In addition to the mass balance, we also have a thermal balance, in which the heat supplied by the fluid and the latent heat released upon crystallization of fresh solid are balanced by the conductive transport of heat into the solid, i.e.

$$
\rho_{s} \mathscr{L} \dot{X}=k_{s} \frac{\partial T_{s}}{\partial n}-k_{l} \frac{\partial T}{\partial n},
$$

where $\mathscr{L}$ is the latent heat released per unit mass of solid. Although the melt may cool rapidly to its liquidus temperature, solidification is still controlled by the requirement that the latent heat released at the solidification front be removed at the cold wall $x=0$. Note that when the temperature at the solid/liquid interface is constrained to be equal to the eutectic temperature, the rate of solidification is not directly influenced by diffusion of mass, but is controlled by the heat balance alone.

\subsection{Dimensionless boundary-layer equations}

The thermal and compositional fluxes out of the fluid depend upon the convective flow adjacent to the advancing solidification front. At large Rayleigh numbers, simple thermally driven single-component convection driven by cooling from one side is characterized by a thin boundary-layer flow sinking parallel to the cold wall (Ostrach 1953). The addition of a second component to the fluid raises the possibility of competition between thermal and compositional effects upon the fluid density, in which an important role is played by the difference in the thermal and compositional diffusivities of the fluid (Nilson 1985).

Before summarizing the results of Nilson (1985), we shall outline the dimensionless boundary-layer equations. This enables us to identify the important dimensionless groups which we use later in determining our scaling laws. Following Nilson (1985), we assume for now that the far-field fluid temperature and composition remain constant, that the solid/liquid interface may be considered vertical $(\partial X / \partial y \ll 1)$, and that horizontal velocities far exceed the solid growth rate $(u \gg \dot{X})$.

We construct (primed) dimensionless variables by writing $\boldsymbol{x}=L \boldsymbol{x}^{\prime} ; t=\left(L^{2} / \kappa_{T l}\right) t^{\prime}$; $\boldsymbol{u}=\left(\kappa_{T l} / L\right) \boldsymbol{u}^{\prime} ; T_{l}=T_{E}+\Delta T T^{\prime} ;$ and $C_{l}=C_{E}+\Delta C\left(C^{\prime}-1\right)$, where $\Delta T=\left(T_{0}-\right.$ $\left.T_{E}\right), \Delta C=\left|C_{0}-C_{E}\right|$, and $\kappa_{T l}=k_{l} / \rho_{l} c_{p l}$. With this rescaling for composition, the dimensionless eutectic composition is 1 , while the dimensionless far-field melt composition $C_{\infty}^{\prime}$ is 0 if $C_{0}<C_{E}$ and 2 if $C_{0}>C_{E}$. The chamber has dimensionless length 1 and dimensionless height $A=H / L$, where $H$ is the chamber height and $L$ is the chamber length. Dropping primes and assuming that horizontal derivatives are much larger than vertical derivatives $\left(\partial_{x} \gg \partial_{y}\right)$, we then obtain from (3.1)-(3.5) the dimensionless boundary-layer equations at the solidification front

$$
\begin{aligned}
\frac{1}{\operatorname{Pr}}\left(u \frac{\partial v}{\partial x}+v \frac{\partial v}{\partial y}\right) & =\frac{\partial}{\partial x}\left(\chi \frac{\partial v}{\partial x}\right)+R a_{T}(T-1)-R a_{C}\left(C-C_{\infty}\right) \\
\left(u \frac{\partial T}{\partial x}+v \frac{\partial T}{\partial y}\right) & =\frac{\partial^{2} T}{\partial x^{2}} \\
\left(u \frac{\partial C}{\partial x}+v \frac{\partial C}{\partial y}\right) & =\frac{1}{L e} \frac{\partial^{2} C}{\partial x^{2}}
\end{aligned}
$$


and

$$
\frac{\partial u}{\partial x}+\frac{\partial v}{\partial y}=0
$$

Here $P r=v_{0} / \kappa_{T l}$ is the Prandtl number and $L e=\kappa_{T l} / \kappa_{C}$ is the Lewis number, where $v_{0}$ is the initial kinematic viscosity of the fluid. The dimensionless fluid viscosity is $\chi=v / v_{0}$. The thermal and compositional Rayleigh numbers, $R a_{T}$ and $R a_{C}$ respectively, are defined by

$$
R a_{T}=\frac{g \alpha \Delta T L^{3}}{v_{0} \kappa_{T l}} \quad \text { and } \quad R a_{C}=\frac{g \beta \Delta C L^{3}}{v_{0} \kappa_{T l}} .
$$

For fluid of constant viscosity and $\operatorname{Pr} \gg 1$, the structure of the laminar boundary layer is determined primarily by the relative direction and magnitude of the compositional and thermal buoyancy anomalies. If compositionally heavy fluid is released at the solidification front, both compositional and thermal buoyancy anomalies drive downflow. However, if compositionally light fluid is released, counterbuoyant convection is possible (Gebhart \& Pera 1971), depending upon the values of the Lewis number $L e$ and of the buoyancy ratio $\Gamma$ (Nilson \& Baer 1982; Nilson 1985), where

$$
\Gamma=\frac{\alpha \Delta T}{\beta \Delta C}=\frac{R a_{T}}{R a_{C}} .
$$

Although the compositional buoyancy anomaly may be larger than the thermal buoyancy anomaly $(\Gamma<1)$, the compositional diffusivity of the fluid is typically smaller than its thermal diffusivity $(L e>1)$, which reduces the range of influence of the compositional forcing. The various asymptotic regimes for $\operatorname{Pr} \gg 1$ and $L e \gg 1$ are presented schematically in figure 3, following Nilson (1985). For $\Gamma L e^{1 / 3} \geq 1$, the boundary-layer flow is thermally dominated and exhibits unidirectional downflow. If $\Gamma L e \lesssim 1$, compositional effects dominate and there is unidirectional upflow. For $L e^{-1} \lesssim \Gamma \lesssim L e^{-1 / 3}$, the boundary layer exhibits counterflow, with the fluid adjacent to the boundary rising and the fluid farther away sinking. Anticipated conditions during magmatic crystallization are also plotted, following Nilson et al. (1985). Note that for fluids such as magma, whose viscosities are strongly dependent upon composition and/or temperature such that they increase close to the solidification front, the boundaries between regimes are shifted (Nilson et al. 1985). For example, if the fluid released at the solidification front is more viscous than the interior fluid, the boundary between the thermally dominated and counterflow regimes (see figure 3 ) is shifted upwards, thus making counterflow more likely.

\section{Finite chamber, constant viscosity}

\subsection{Numerical calculations}

Before evaluating their numerical solution, the transport equations (3.1)-(3.3) were non-dimensionalized, as described in $\$ 3.2$, and the curl taken of the momentum equation (3.1) to eliminate the pressure term. We solved for the flow field using a streamfunction-vorticity formulation, similar to that described by Thompson \& Szekely (1988). Further details of the numerical method are outlined in the Appendix. Note that the dimensionless form of the interfacial heat balance introduces an additional dimensionless group, the Stefan number,

$$
S t=\frac{\kappa_{T l}}{\kappa_{T s}} \frac{\mathscr{L}}{c_{p s} \Delta T},
$$




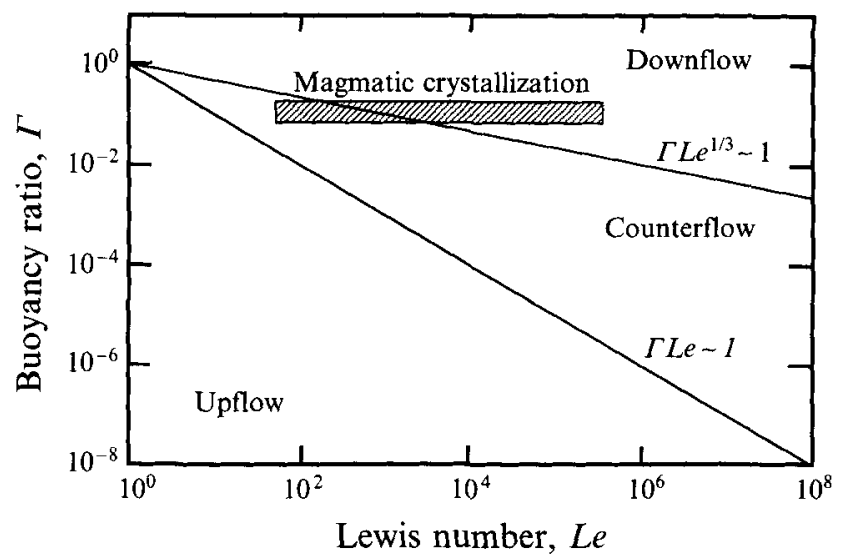

FiguRE 3. Parameter ranges for thermally dominated downflow, compositionally-dominated upflow and counterflow in a double-diffusive boundary layer adjacent to a vertical boundary. The shaded box labelled crystallization represents the range of values typical of solidification in a magma chamber (after Nilson 1985).

which describes the importance of latent heat release at the solid/liquid interface. The thermal diffusivity of the solid phase is $\kappa_{T s}=k_{s} / \rho_{s} c_{p s}$.

For illustration, the convective flow field and fluid composition from a typical calculation are presented in figure 4. The initial fluid composition is supereutectic $\left(C_{0}>C_{E}\right)$, which means that the fluid adjacent to the solid/liquid interface is colder than the bulk interior fluid, but also relatively depleted in the heavier component. Initially, the buoyancy ratio $\Gamma=0.14$ and the Lewis number $L e=32.5$. Hence, in steady state, we expect counterflow in the double-diffusive boundary layer. The Rayleigh numbers are $R a_{C}=3.9 \times 10^{9}$ and $R a_{T}=5.4 \times 10^{8}$. During the initial transient $(t \lesssim 0.003)$ before the steady-state flow becomes established, convection is thermally dominated (figure $4 a$ ) and flow is counterclockwise everywhere, with the exception of small, compositionally dominated regions adjacent to the solidification front at the top and bottom of the domain. The lower compositionally dominated region grows until there is a clearly visible clockwise-flowing compositional convection cell. As the alloy continues to solidify, the compositionally dominated convection cell grows at the expense of the thermal cell, until the fluid essentially loses all superheat (figure $4 k$ ).

After the thermally dominated initial transient has passed, the compositionally light fluid rises up the solidification front and intrudes into the interior to form a compositionally stratified layer at the roof (figure $4 h$ ). This layer continues to grow until the entire fluid region is compositionally stratified (figure $4 l$ ). As solidification continues, the fluid composition evolves towards the eutectic at all levels. This process is termed laminar box-filling, and has been studied experimentally and theoretically by Worster \& Leitch (1985). The flow reversal observed in the top-right-hand corner of figure $4(i)$ is a typical feature of free convection in a stratified fluid (Worster \& Leitch 1985).

The composition of the solid product (see figure 5) reflects the evolution of the fluid flow and composition fields. Much of the following explanation has also been provided by Leitch $(1985,1987,1990)$ and Huppert et al. (1987), who observed similar patterns of compositional stratification in their experiments with aqueous sodium carbonate solutions. Let us consider a horizontal slice at any level. Moving 

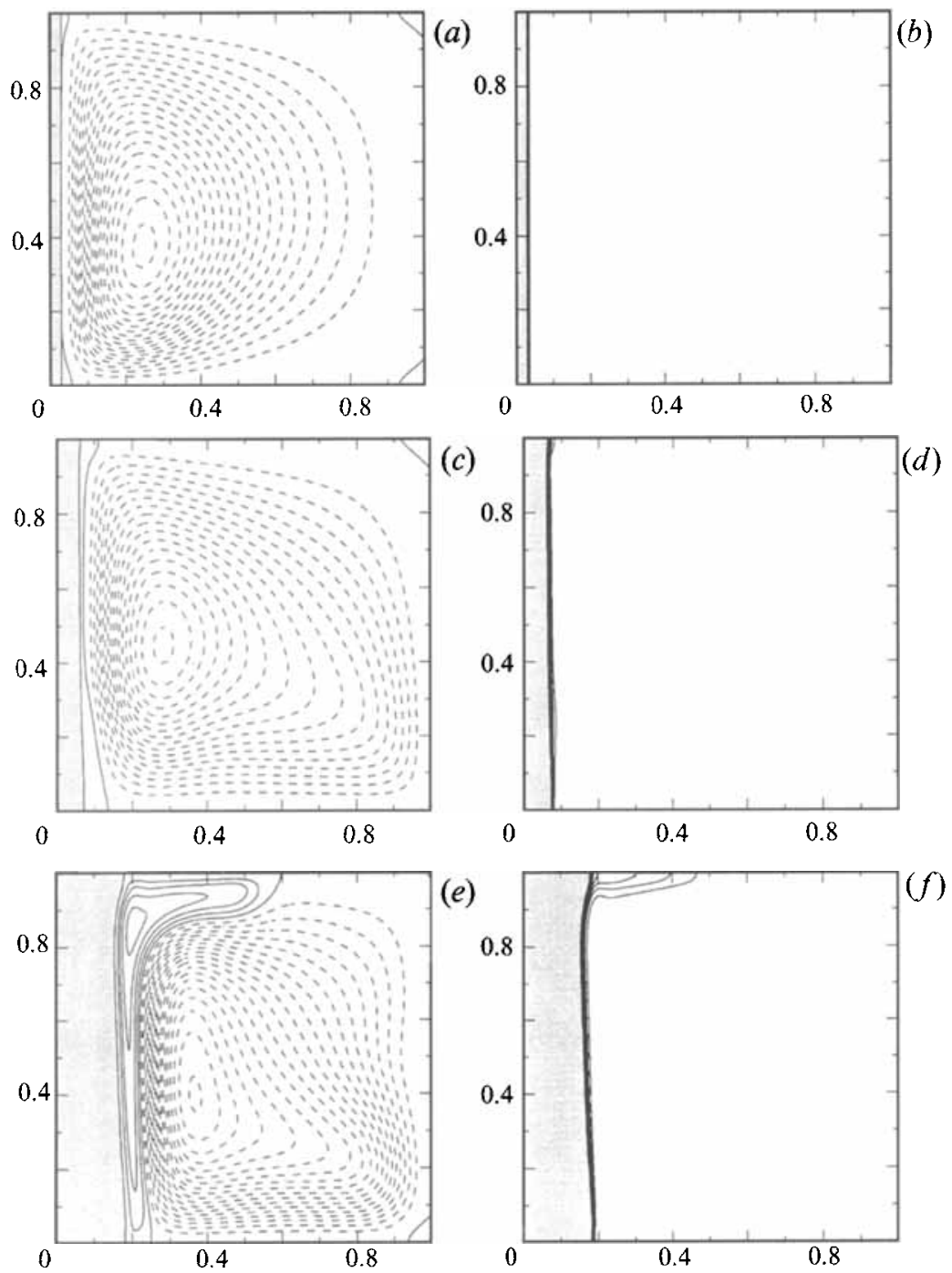

Figure $4(a-f)$. For caption see facing page.

away from the cold boundary $(x=0)$, the solid composition first increases, away from the eutectic. This is because the diffusive flux of composition across the quasi-steadystate compositional boundary layer remains approximately constant at a given height, with the diffusive flux away from the interface balanced by advection of composition towards the solidification front, while the solidification rate continues to decrease $\left(\dot{X} \propto t^{-1 / 2}\right)$. Hence, from equation (3.7), the solid composition increases. However, at later times, laminar box filling leads to a gradual decrease in the composition of the interior fluid, starting from the roof down. This reduces the compositional flux into the solid, and the solid composition now decreases towards eutectic. The position of the maximum composition at a given height corresponds to the time at which the base of the stratified layer reaches that level.

From any vertical slice, we see that there is a further compositional stratification related to the thickening of the upflowing compositional boundary layer with height 

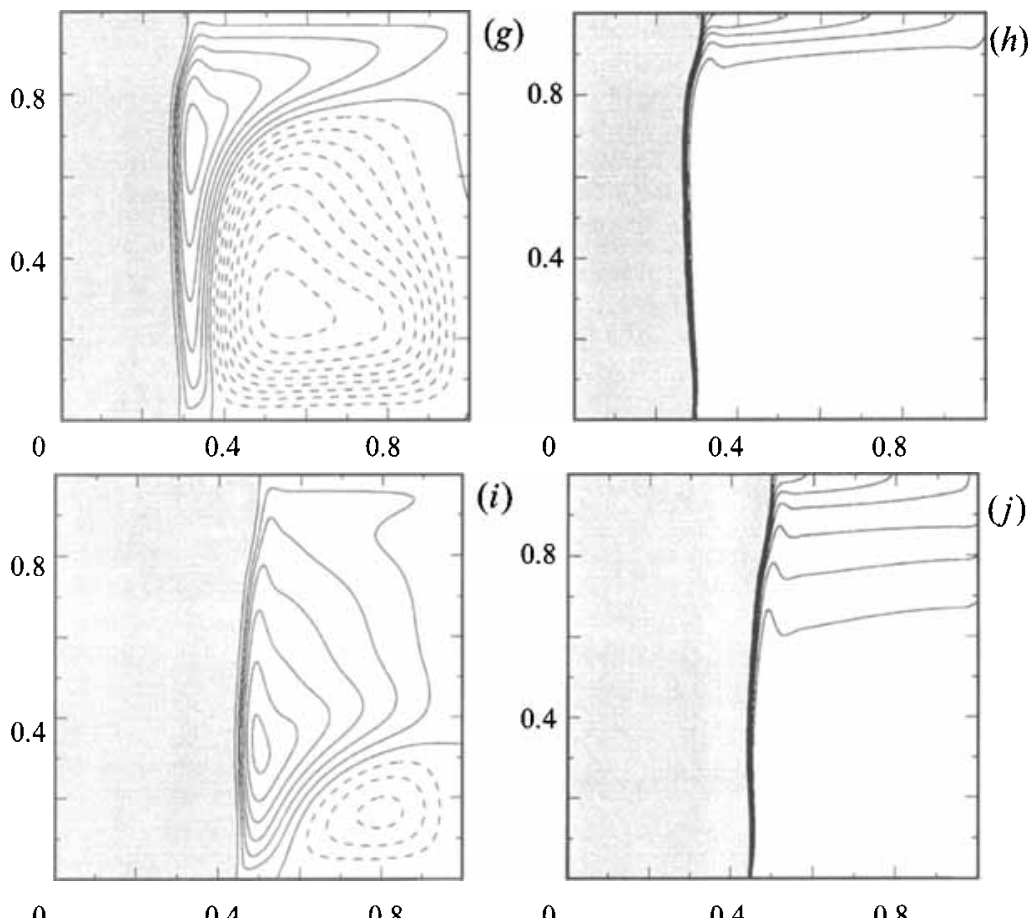

(i)
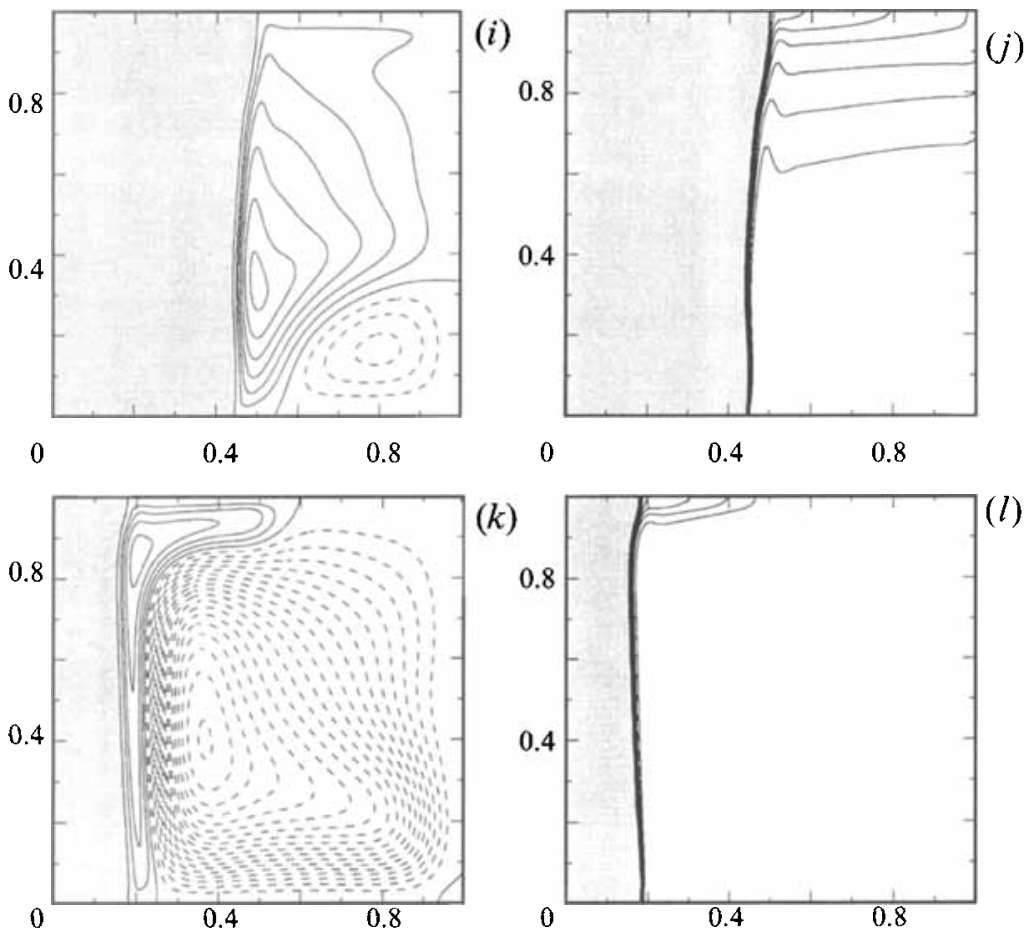

FIGURE 4. A typical complete freezing calculation for a supereutectic melt with light fluid release at the solidification front. Dimensional values of parameters are listed in table 1 and $\$ 5.2$. Contours of the fluid streamfunction (above) and composition (see facing page) are plotted after dimensionless times: $(a, b) t=0.002 ;(c, d) t=0.008 ;(e, f) t=0.032 ;(g, h) t=0.064 ;(i, j) t=0.128$; and $(k, l) t=0.256$. The contour interval for the streamfunction is 2 for $(a)$ and $(c)$, and 0.5 for the remainder. Negative values (including zero), which represent clockwise flow, are contoured using solid lines, and positive values are represented by dashed lines, thereby emphasizing the compositionally-driven convection cell over the thermally driven cell. The contour interval for composition is 0.05 . At early times, the flow is thermally dominated and anticlockwise. At later times, the flow is compositionally dominated and clockwise.

above the floor of the chamber. At the base of the chamber, the compositional flux across the boundary layer is greater than at the roof for a given interior composition, which means that the solid composition decreases with height. This effect is exacerbated at later times by the compositional stratification in the fluid produced by box filling. Consequently, the maximum solid composition occurs at the base of the solid, and its position with respect to the cooling boundary is determined 


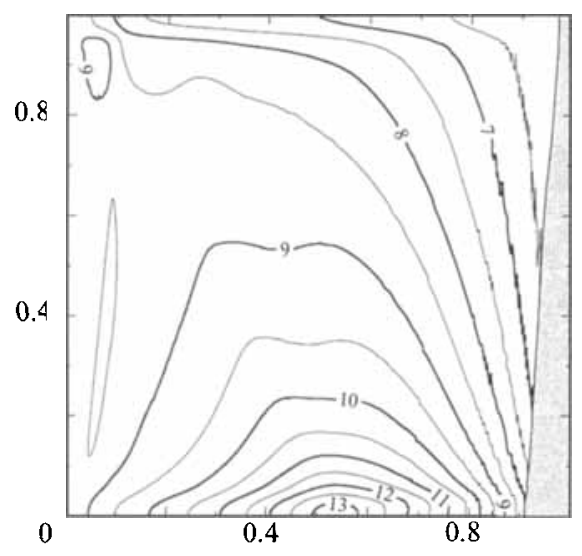

FIGURE 5. Composition of the solid for the calculation shown in figure 4. Contours are labelled in wt $\% \mathrm{Na}_{2} \mathrm{CO}_{3}$ for comparison with the experimental data of Leitch (1985). Dimensionless composition 1 would correspond to $6.0 \mathrm{wt} \%$, and the maximum solid composition is about $13.2 \mathrm{wt} \%$ at the base of the tank, at $x=0.53$. The cold wall is at $x=0$.

by the position of the solidification front when the base of the stratified layer reaches the bottom of the chamber.

The picture is slightly complicated by the vertical dependence of the solid thickness. This results from vertical variations in the thermal flux across the combined thermal and compositional boundary layer. However, for most of the calculation, the solid/liquid interface remained approximately vertical, and the convection close to the interface was compositionally dominated. The compositional stratification in the solid is dominated in this example by the compositional convection, and the effects of thermal convection are secondary.

\subsection{Comparison of laminar box filling and solidification timescales}

The experiments of Leitch $(1985,1987)$ and Huppert et al. (1987), and the numerical calculations described above, suggest that the pattern of compositional stratification in the solid is determined by the ratio of the timescales for solidification and for laminar box filling by the compositionally light fluid, provided that convection is compositionally dominated in the vicinity of the solidification front.

To determine the solidification timescale, we assume that the heat flux from the melt is negligible, and that the rate of solidification is controlled by the balance between latent heat release at the solid/liquid interface and thermal conduction through the solid. If the fluid is initially above its liquidus temperature, this approximation may only be valid after most of the superheat in the chamber has been removed, and we shall return to this point later.

For sufficiently slow solidification, the temperature profile in the solid is approximately linear, and from the dimensionless form of equation (3.9)

$$
\text { St } \dot{X} \sim \frac{1}{X}
$$

which implies a solidification timescale

$$
t_{S} \sim S t
$$

The rate at which the stratified fluid region fills the chamber is determined by the 
upward flux of light fluid in the compositional boundary layer at the approximately horizontal interface between the light, fractionated fluid and the relatively heavy bulk fluid. If $L e \gg 1$ and $\Gamma L e^{1 / 3} \lesssim 1$, the compositional boundary layer is embedded deep within the thermal boundary layer and has thickness (Nilson 1985)

$$
\delta_{C} \sim\left(\frac{y}{L e R a_{C}}\right)^{1 / 4},
$$

where $y$ is measured in the downstream direction for the inner compositional boundary layer flow. The magnitude of the upward velocity is

$$
v \sim\left(\frac{y R a_{C}}{L e}\right)^{1 / 2} .
$$

Therefore, following Worster \& Leitch (1985), the timescale for box filling is

$$
t_{F} \sim \frac{A}{v \delta_{C}} \sim A^{1 / 4} L e^{3 / 4} R a_{C}^{-1 / 4},
$$

provided that the volume of the fluid region remains approximately constant (i.e. $\left.t_{F} \ll t_{S}\right)$. The ratio of (4.6) and (4.3),

$$
\frac{t_{F}}{t_{S}} \sim A^{1 / 4} L e^{3 / 4} R a_{C}^{-1 / 4} S t^{-1},
$$

then describes the relative rates of box filling and solidification.

If $t_{F} \sim t_{S}$, the ratio of timescales would still scale as in equation (4.7), but would be shorter by a factor dependent upon $t_{F} / t_{S}$ itself. However, if $t_{F} \gg t_{S}$, which is the limit of rapid solidification, box filling will occur over the solidification timescale as a result of the rapid reduction in the size of the fluid region at late stages of solidification.

An analogous expression to equation (4.6) may be derived for the timescale for box filling by colder fluid which sinks in the outer part of the boundary layer and fills the chamber from the bottom. This thermal box-filling timescale is smaller than the compositional box-filling timescale by a factor $\Gamma^{1 / 4} L e^{3 / 4} \gg 1$ when there is an inner compositionally driven boundary layer $(\Gamma L e \gtrsim 1, L e \gg 1)$. Under these circumstances the thermal flux from the fluid may indeed be neglected over the compositional box-filling timescale.

The ratio (4.7) describes the magnitude of the compositional stratification in the solid, as well as its pattern. The dimensionless flux of composition from the fluid into (or out of) the solid at the solid/liquid interface is given by

$$
F_{C} \sim \frac{1}{L e} \frac{\left(C_{i}-C_{E}\right)}{\delta_{C}},
$$

where $C_{i}(X, y / A)$ is the far-field composition at height $y$ when the solidification front is at $x=X$, and which is also determined by $t_{F} / t_{s}$. Now, from equation (3.7), we see that the difference $\Delta C_{s}=\left|C_{s}-C_{E}\right|$ between the solid composition and the eutectic composition is

$$
\Delta C_{s} \sim \frac{F_{C}}{\dot{X}} .
$$

Therefore, substituting from (4.2) for $\dot{X}$ and (4.4) for $\delta_{C}$, we obtain

$$
\frac{\Delta C_{s}}{\left|C_{i}-C_{E}\right|} \sim \frac{X}{y^{1 / 4}} L e^{-3 / 4} R a_{C}^{1 / 4} S t .
$$


Equation (4.10) confirms that, before box filling occurs, the absolute difference between the solid composition and the eutectic composition increases with distance away from the cold wall and decreases in the downstream direction, as observed in the experiments of Leitch $(1985,1987)$ and Huppert et al. (1987), and in the numerical calculations reported above. As the fractionated fluid gradually fills the chamber, the compositional difference between the far-field fluid and the fluid at the solid/liquid interface, $\left|C_{i}-C_{E}\right|$, is reduced and the solid composition approaches the eutectic $\left(\Delta C_{s} \rightarrow 0\right)$. The widening of the compositional boundary layer in the downstream direction leads to vertical stratification in the solid even when the bulk fluid is homogeneous. The solid towards the base of the chamber becomes enriched in the heavy component, regardless of the direction of boundary-layer flow, as long as it is compositionally dominated or counterflowing.

The above scaling analysis has important implications for the modelling of experiments and natural systems characterized by Rayleigh numbers and Lewis numbers too high for present-day computational resources. Laboratory experiments at moderate Lewis and Rayleigh numbers $\left(L e \sim 10^{2}-10^{3}, R a_{C} \sim 10^{10}-10^{11}\right)$ can be performed to study the solidification of magma chambers at much higher values $\left(\right.$ Le $\sim 10^{4}-10^{6}$, $R a_{C} \sim 10^{15}-10^{19}$ ), and numerical calculations performed at even lower Lewis and Rayleigh numbers may be employed to model both (see figure 6), provided that the combination of parameters

$$
P \sim A^{1 / 4} L e^{3 / 4} R a_{C}^{-1 / 4} S t^{-1}
$$

is the same in all three cases, and provided that our model assumptions remain valid. Larger values of $P$ correspond to relatively slow box filling, owing to rapid solidification $(S t \ll 1)$, weak convection, a thin compositional boundary layer ( $L e \gg$ $1)$, or a tall chamber geometry $(A \gg 1)$. Under these circumstances, the fluid is largely homogeneous and the compositional stratification in the solid is determined by boundary-layer thickening and by the gradual reduction in the growth rate of the solid. For $P \ll 1$, box filling is rapid and the fluid compositional gradually approaches the eutectic as solidification proceeds. The solid composition reflects the fluid composition and approaches the eutectic as one moves away from the cold wall. In the following section we test our scaling analysis by simulating experiments reported by Leitch $(1985,1987)$ and Huppert et al. (1987).

Before proceeding, however, we note two caveats. First, the above scalings are valid only if the solid growth rate $\dot{X} \ll u$, the horizontal velocity in the compositional boundary layer. Otherwise, the structure of the compositional boundary-layer flow is determined by a balance between the rates of solidification and compositional diffusion. The incompressibility condition (3.13) implies that $u \sim v \delta_{C} / y$, and the solidification rate $\dot{X} \sim(\text { St } X)^{-1}$. Hence, substituting from (4.4) and (4.5) for $\delta_{C}$ and $v$ respectively, we require $P \ll X$ for the above analysis to be valid. Quantitative agreement between experiments and numerical calculations should therefore only be expected when $P \ll 1$, although the qualitative description of the pattern of compositional stratification in terms of $t_{F} / t_{S}$ remains appropriate. Second, the ratio of thermal to compositional box-filling timescales is preserved under the above rescaling, but increases when the Lewis number is reduced. This means that the duration of thermal convection will be extended in the numerical calculations, which explains why in the above model calculation a small thermal convection cell remained until solidification was almost complete. 


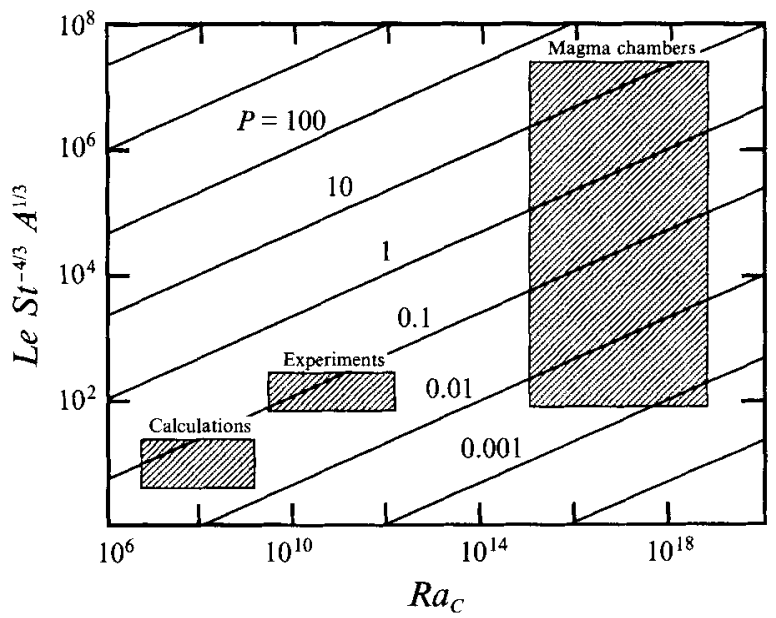

FIGURE 6. Contours of the parameter $P$, as defined by equation (4.11), for a range of values of the compositional Rayleigh number $R a_{C}$ and $L e S t^{-4 / 3} A^{1 / 3}$, where $L e$ is the Lewis number, $S t$ is the Stefan number and $A=H / L$ is the aspect ratio of the chamber. The parameter $P$ represents the ratio of timescales for laminar box filling and for solidification. Small values of $P$ correspond to rapid box-filling rates or slow solidification, and result in stronger vertical compositional stratification in the solid. Shaded areas represent areas typical of magma chambers (dimension $\sim 1 \mathrm{~km}$ ), the laboratory experiments of Leitch $(1985,1987)$ and Huppert et al. (1987), and the numerical calculations presented in this paper.

\section{Comparison with laboratory experiments}

A series of laboratory experiments were performed by Leitch $(1985,1987)$ and Huppert et al. (1987) to study the compositional stratification in a binary alloy cooled from one side. Aqueous sodium carbonate solutions were completely frozen in Perspex chambers, approximately $20 \mathrm{~cm}$ long each side, by cooling from one sidewall, with all other boundaries insulated. Each freezing experiment took on the order of 24 hours, after which the solid crystalline block was cut up into pieces and the composition of each piece measured.

The morphology of the solid/liquid interface during the experiments depended chiefly upon the major component crystallizing out. For subeutectic solutions, the major crystalline product was ice, and the solidification front was fairly smooth. However, for supereutectic solutions, large, faceted hydrated sodium carbonate $\left(\mathrm{Na}_{2} \mathrm{CO}_{3} \cdot 10 \mathrm{H}_{2} \mathrm{O}\right)$ crystals formed, and the solid/liquid interface was distinctly irregular (Leitch 1985). Nevertheless, for the supereutectic experiments the boundary region across which the larger crystals protruded was only of the order of millimetres thick (Leitch 1987), with the exception of one set of experiments reported by Huppert et al. (1987), in which a thick mush formed at the base of an initially stably stratified tank.

To make a comparison between these experiments and the above scaling analysis, we select two examples for which most data about the composition of the solid have been published. Both come from Leitch (1985), although the supereutectic experiment is also described by Huppert et al. (1987) and Leitch (1990). Similar features in experiments with metal alloys have also been described by Hebditch (1975). Values for the physical constants, which are presented in table 1, suggest that the compositional Rayleigh numbers for the two experiments were approximately $2.7 \times 10^{9}$ and $3.9 \times 10^{9}$ respectively, and the Lewis number $L e \sim 330$ in both cases. 


\begin{tabular}{cccl} 
Quantity & Aqueous $\mathrm{Na}_{2} \mathrm{CO}_{3}$ solution & Magma chamber & \multicolumn{1}{c}{ Units } \\
$c_{p l}$ & $3.9 \times 10^{3}$ & $1.3 \times 10^{3}$ & $\mathrm{~J} \mathrm{~kg}^{-1}{ }^{\circ} \mathrm{C}^{-1}$ \\
$c_{p s}$ & $1.9 \times 10^{3}$ & $1.3 \times 10^{3}$ & $\mathrm{~J} \mathrm{~kg}^{-1}{ }^{\circ} \mathrm{C}^{-1}$ \\
$\kappa_{T l}$ & $1.3 \times 10^{-7}$ & $5.0 \times 10^{-7}$ & $\mathrm{~m}^{2} \mathrm{~s}^{-1}$ \\
$\kappa_{T s}$ & $9.0 \times 10^{-7}$ & $5.0 \times 10^{-7}$ & $\mathrm{~m}^{2} \mathrm{~s}^{-1}$ \\
$v$ & $2.3 \times 10^{-6}$ & $10^{-2}-10$ & $\mathrm{~m}^{2} \mathrm{~s}^{-1}$ \\
$\kappa_{C}$ & $4.0 \times 10^{-10}$ & $10^{-9}-10^{-12}$ & $\mathrm{~m}^{2} \mathrm{~s}^{-1}$ \\
$\rho_{0}$ & $1.0 \times 10^{3}$ & $2.5 \times 10^{3}$ & $\mathrm{~kg} \mathrm{~m}^{-3}$ \\
$\mathscr{L}$ & $3.1 \times 10^{5}$ & $5.0 \times 10^{5}$ & $\mathrm{~J} \mathrm{~kg}^{-1}$ \\
$\alpha$ & $2.0 \times 10^{-4}$ & $2.5 \times 10^{-5}$ & ${ }^{\circ} \mathrm{C}^{-1}$ \\
$\beta$ & $1.0 \times 10^{-2}$ & & $\left(\mathrm{wt} \% \mathrm{Na}_{2} \mathrm{CO}_{3}\right)^{-1}$ \\
$\Delta \rho_{C}$ & & $2.5 \times 10^{2}$ & $\mathrm{~kg} \mathrm{~m}^{-3}$
\end{tabular}

TABLE 1. Physical constants for aqueous sodium carbonate system at $0^{\circ} \mathrm{C}$, obtained from various sources, including Leitch (1985), Woods \& Huppert (1989) and Weast (1971), and for magma chambers (Nilson et al. 1985). For the magma chamber, a characteristic density change $\Delta \rho_{C}$ is listed rather than $\beta$, the coefficient of expansion due to variations in composition. An equivalent value for $\beta$ is given by $\Delta \rho_{c} / \rho_{0} \Delta C$.

For each numerical simulation, we divided the experimental values of the Rayleigh numbers by a factor of $10^{3}$ and the Lewis numbers by a factor of 10 , in accordance with the above scaling analysis.

\subsection{Subeutectic solution}

In this experiment, the fluid initially had uniform temperature $T_{0}=22{ }^{\circ} \mathrm{C}$ and composition $C_{0}=4.0 \mathrm{wt} \% \mathrm{Na}_{2} \mathrm{CO}_{3}$ (for an aqueous sodium carbonate solution, $C_{E}=5.98 \mathrm{wt} \% \mathrm{Na}_{2} \mathrm{CO}_{3}, T_{E}=-2.1^{\circ} \mathrm{C}$ ). The cold wall temperature was reduced from $-10{ }^{\circ} \mathrm{C}$ to $-36{ }^{\circ} \mathrm{C}$ over a period of about 5 hours (Leitch 1985), and this was explicitly accounted for in the numerical calculations. Contours of solid composition are shown in figure 7 for a slice taken in the $(x, y)$-plane, midway between the two endwalls. For a subeutectic solution, the fluid released at the solid/liquid interface is enriched in the heavy component $\left(\mathrm{Na}_{2} \mathrm{CO}_{3}\right)$, and therefore sinks. Box filling of fractionated fluid occurs from the floor of the chamber upwards and there is a minimum in solid composition $\left(2.0 \mathrm{wt} \% \mathrm{Na}_{2} \mathrm{CO}_{3}\right)$ approximately $12 \mathrm{~cm}$ from the cold boundary, at the roof of the tank.

To simulate this experiment, it was first necessary to neglect the thermal flux from the fluid. Leitch (1985) reports that initially, in the experiment, there was no solid growth towards the top of the cold boundary, owing to the advection of warm fluid towards the boundary, and that growth only commenced there when the fluid superheat had been substantially reduced. However, equation (3.9) always implies solid growth at early times, whatever the thermal flux from the fluid, because the heat flux through the solid is initially infinite. In response to the large thermal flux, the solid simply grows very slowly, thus creating, close to the cold boundary, a strong minimum in solid composition which is an artefact of the crystallization model (Jarvis 1991). Therefore, in this particular numerical calculation, thermal effects in the fluid have been removed entirely by setting the thermal Rayleigh number to zero, and by neglecting the heat flux from the melt in equation (3.9).

The calculated solid composition for the above experimental conditions is presented in figure 8 . The calculation was stopped when only $93 \%$ of the alloy had solidified because convergence of the numerical scheme was becoming very slow. The final 


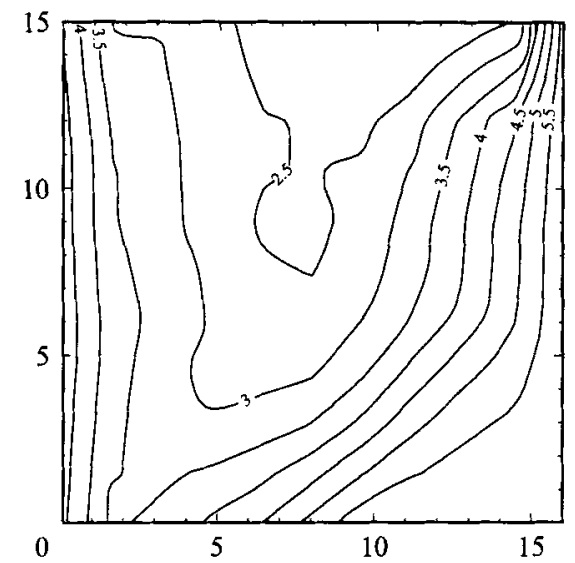

FIGURE 7. Contour plot of the solid composition for a complete freezing experiment with a homogeneous subeutectic aqueous sodium carbonate solution (from original data kindly provided by A.M. Leitch). The contours are labelled in $w t \% \mathrm{Na}_{2} \mathrm{CO}_{3}$. The solid composition was measured by cutting the solid up into sections, allowing them to melt and measuring the refractive index of each sample. The solid block has length $16 \mathrm{~cm}$ and depth $15 \mathrm{~cm}$.

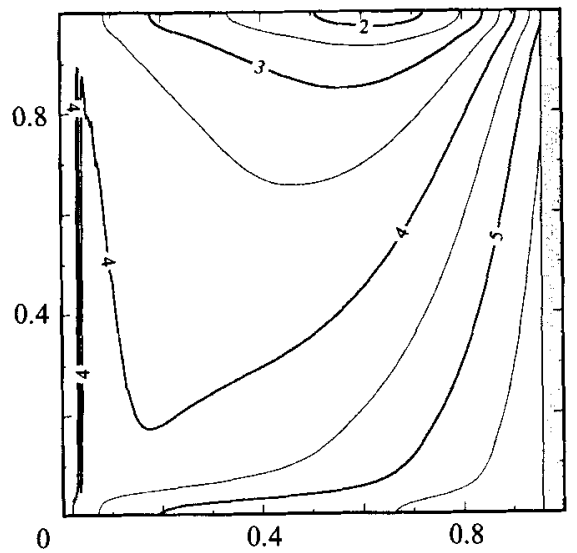

FIGURE 8. Calculation of the complete freezing of a homogeneous subeutectic aqueous sodium carbonate solution. Thermal effects in the fluid have been removed by setting $R a_{T}=0$ and by neglecting the heat flux from the liquid. As far as possible, physical conditions were taken from Leitch (1985) (see table 1), although the Rayleigh numbers were decreased by a factor of $10^{3}$ and the Lewis number by a factor of 10 , in accordance with the scaling analysis presented in $\$ 4.2$. Contours labelled as for figure 5 .

solid formed would have had eutectic composition. The pattern of compositional stratification is very similar to the experimental result (figure 7), with a minimum composition of $1.8 \mathrm{wt} \% \mathrm{Na}_{2} \mathrm{CO}_{3}$ just over $10 \mathrm{~cm}$ from the cold wall. Note that, even if the experiments were modelled precisely, the minimum solid composition calculated would still be below the minimum measured composition because finite-sized blocks, with sides of approximately $2 \mathrm{~cm}$, were sampled in measuring the composition of the solid grown in the laboratory. This numerical calculation may be considered strong support for the scaling arguments outlined in $\$ 4$. 


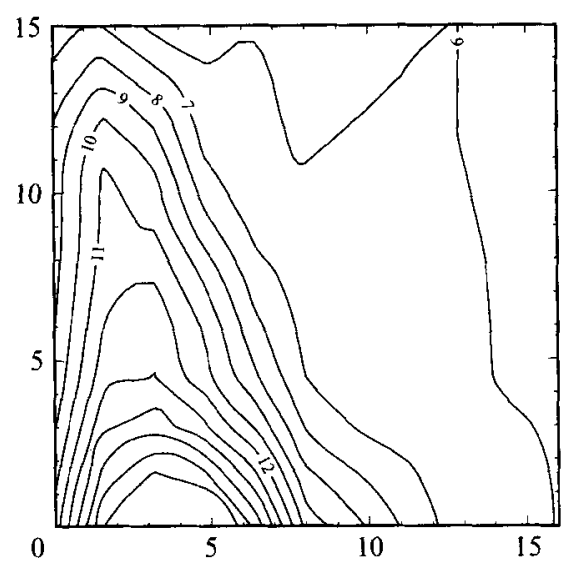

Figure 9. Contour plot of the solid composition for a complete freezing experiment with a homogeneous supereutectic aqueous sodium carbonate solution (from original data kindly provided by A.M. Leitch). The contours are labelled in wt $\% \mathrm{Na}_{2} \mathrm{CO}_{3}$ and the solid block has length $16 \mathrm{~cm}$ and depth $15 \mathrm{~cm}$.

\subsection{Supereutectic solution}

In the second experiment, the initial temperature $T_{0}=18{ }^{\circ} \mathrm{C}$, the initial composition $C_{0}=8.8 \mathrm{wt} \% \mathrm{Na}_{2} \mathrm{CO}_{3}$, and the cold boundary temperature was reduced from $-10{ }^{\circ} \mathrm{C}$ to $-36{ }^{\circ} \mathrm{C}$ over a period of about 2 hours (Leitch 1985). The measured solid composition, also taken midway between the two endwalls, is shown in figure 9. The maximum solid composition is about $17.5 \mathrm{wt} \% \mathrm{Na}_{2} \mathrm{CO}_{3}$, approximately $4 \mathrm{~cm}$ from the cold boundary at the base of the tank.

The corresponding numerical simulation is the one described previously in $\S 4.1$. Figure 4 shows the evolution of the flow field and fluid composition, while contours of solid composition are presented in figure 5. Again there is a strong qualitative agreement between experiment and calculation, but in constrast to the subeutectic case, the quantitative agreement is quite poor. The calculation places the maximum in solid composition too far from the cold boundary, about $8.5 \mathrm{~cm}$ away, and the maximum composition is only about $13.2 \mathrm{wt} \% \mathrm{Na}_{2} \mathrm{CO}_{3}$.

The most likely reason for the quantitative disagreement is the assumption of laminar flow in the compositional boundary layer. Leitch $(1985,1987)$, in describing the same experiments, notes that there is a large disparity between the box-filling time observed in the laboratory and that predicted by classical laminar boundary-layer theory, and suggests that in this experiment the boundary-layer flow was not laminar, but mildly turbulent, owing to the release of small, compositionally buoyant plumes from the upper surfaces of protruding $\mathrm{Na}_{2} \mathrm{CO}_{3} \cdot 10 \mathrm{H}_{2} \mathrm{O}$ crystals. This increases the upward flux of fractionated fluid, thereby shortening the box-filling timescale.

Comparison between experiments and theory therefore provides mixed results. While the scaling theory is supported where experimental conditions are favourable (smooth, planar solidification front), albeit over a rather small range of Lewis and Rayleigh numbers, it is apparent that the morphology of the solid/liquid interface has a strong role to play under less favourable circumstances, such as the supereutectic aqueous sodium carbonate freezing experiments described above. 


\section{Finite chamber, variable viscosity}

We now extend the scaling theory of $\S 4$ to fluids whose viscosity is strongly dependent upon their temperature and composition. We restrict ourselves to lowbuoyancy-ratio, high-Prandtl-number, high-Lewis-number systems, for which the thermal boundary layer is embedded deep within the viscous boundary layer, and the compositional boundary layer deep within the thermal boundary layer. We shall demonstrate that it is possible to determine a suitable effective viscosity which describes both the rate of growth of the stratified layer of fractionated fluid by box filling, and the pattern and magnitude of compositional stratification in the solid.

\subsection{Structure of the inner compositional boundary layer}

For $\operatorname{Pr} \gg 1$ and $L e \gg 1$ (provided that $\Gamma L e^{1 / 3} \lesssim 1$ ), a similarity solution exists for the inner compositional boundary layer, within which the dominant balance is between viscous forces and buoyancy forces (Kuiken 1968; Spera et al. 1982; Nilson et al. 1985). If we write

$$
\delta(y)=\left(\frac{4 y \tilde{\chi}}{L e R a_{C}}\right)^{1 / 4} \text { and } V(y)=\left(\frac{4 y R a_{C}}{L e \tilde{\chi}}\right)^{1 / 2},
$$

and define the similarity variable, $\eta$, and streamfunction, $\psi$, by

$$
\eta=x / \delta \quad \text { and } \quad \psi=V \delta f(\eta),
$$

then the boundary-layer equations reduce to the ordinary differential equations

$$
\begin{aligned}
\left(\frac{\chi}{\tilde{\chi}} f^{\prime \prime}\right)^{\prime}+C & =2 \\
C^{\prime \prime} & =3 f C^{\prime},
\end{aligned}
$$

with boundary conditions

$$
f(0)=f^{\prime}(0)=0 ; \quad C(0)=1
$$

and

$$
f^{\prime \prime}(\infty)=0 ; \quad C(\infty)=2,
$$

in writing which we have assumed that the melt is supereutectic $\left(C_{0}>C_{E}\right)$; however, the following results apply equally to subeutectic melts. The boundary condition $f^{\prime \prime}(\infty)=0$ was first derived by Kuiken (1968), and is applicable to high-Prandtlnumber, high-Lewis-number fluids. In equation (6.3), $\tilde{\chi}=\tilde{\mu} / \mu_{0}$ is the dimensionless viscosity at the outer edge of the compositional boundary layer, and represents the viscosity variation across the thermal boundary layer alone. Within the inner compositional boundary layer, the dimensionless temperature is equal to the boundary temperature $(T=0)$, and $\chi / \tilde{\chi}$ is a function of composition only.

Typical solutions of $(6.3)-(6.6)$ are presented in figure 10 , for the simple exponential viscosity law

$$
\chi=\tilde{\chi}\left(\frac{\chi_{w}}{\tilde{\chi}}\right)^{2-C},
$$

which corresponds to a dimensionless viscosity $\chi_{w}$ at the vertical boundary $\eta=0$. The similarity solutions are the same as those obtained by Spera et al. (1982) and Nilson et al. (1985), and are calculated using the Runge-Kutta method, combined with a Newton-Raphson shooting method to determine the unknown conditions $f^{\prime \prime}(0)$ and $C^{\prime}(0)$. 

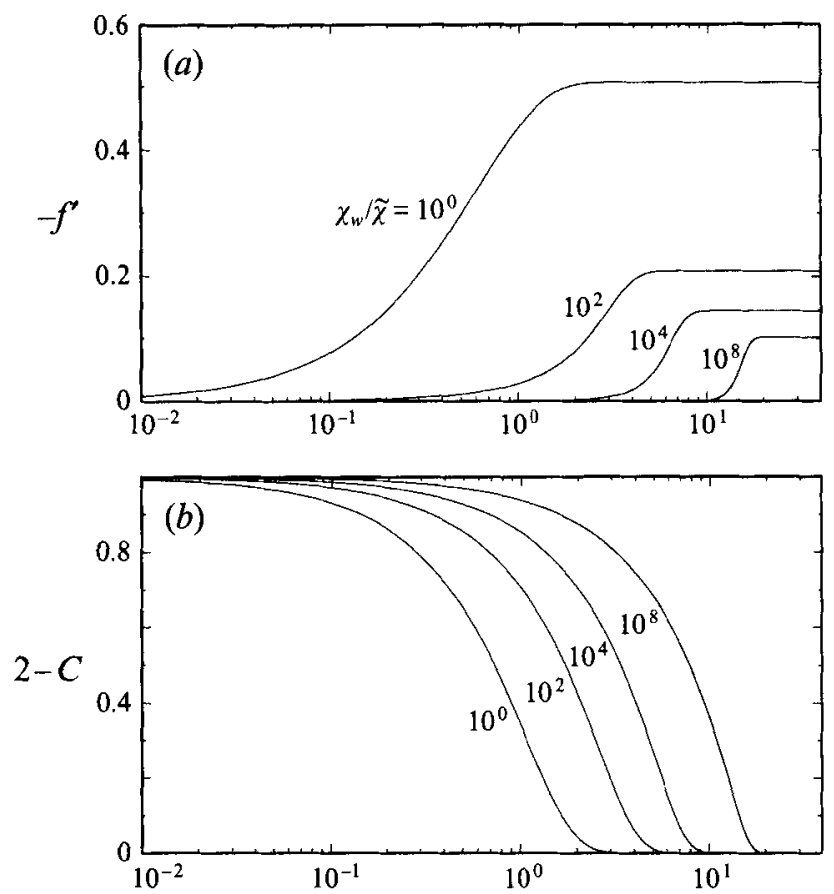

FIGURE 10. Typical similarity solutions for the inner compositional boundary layer for a number of different viscosity variations $\chi_{w} / \tilde{\chi}$. In $(a)$, the upward velocity $-f^{\prime}(\eta)$ is plotted, and in $(b)$ we show the composition anomaly, $2-C(\eta)$. The effect of increasing the viscosity contrast is to weaken the upflow and to move it away from the vertical boundary, $\eta=0$.

A suitable effective viscosity for variable-viscosity convection is the viscosity at which the same total upward compositional flux would be achieved if the fluid viscosity were constant. From equations (6.3) and (6.4), the total upward compositional flux is

$$
F_{U}=-V \delta \int_{0}^{\infty}(2-C) f^{\prime} \mathrm{d} \eta=\frac{1}{3} V \delta C^{\prime}(0),
$$

and by conservation of mass, the compositional flux into the solid is simply

$$
F_{C}=-\frac{\mathrm{d} F_{U}}{\mathrm{~d} y} .
$$

Hence, from the definition (6.1) for $\delta$ and $V$, we find that both the box-filling rate and the solid composition, $\Delta C_{s}$, are proportional to $\left(R a_{C} / \chi\right)^{1 / 4}$. Therefore, if $C_{0}^{\prime}$ is the value of $C^{\prime}(0)$ when $\chi_{w} / \tilde{\chi}=1$, the effective viscosity should be

$$
\chi_{e f f}=\tilde{\chi}\left(\frac{C^{\prime}(0)}{C_{0}^{\prime}}\right)^{4},
$$

and the effective Rayleigh number $R a_{C_{e f f}}=R a_{C} / \chi_{\text {eff }}$. It is useful to write the effective viscosity, $\chi_{e f f}$, as a power law,

$$
\chi_{e f f}=\tilde{\chi}\left(\frac{\chi_{w}}{\tilde{\chi}}\right)^{\gamma},
$$

where the variable exponent satisfies $0<\gamma<1$. When $\gamma$ is small, the increase in viscosity associated with compositional variations close to the wall is relatively 

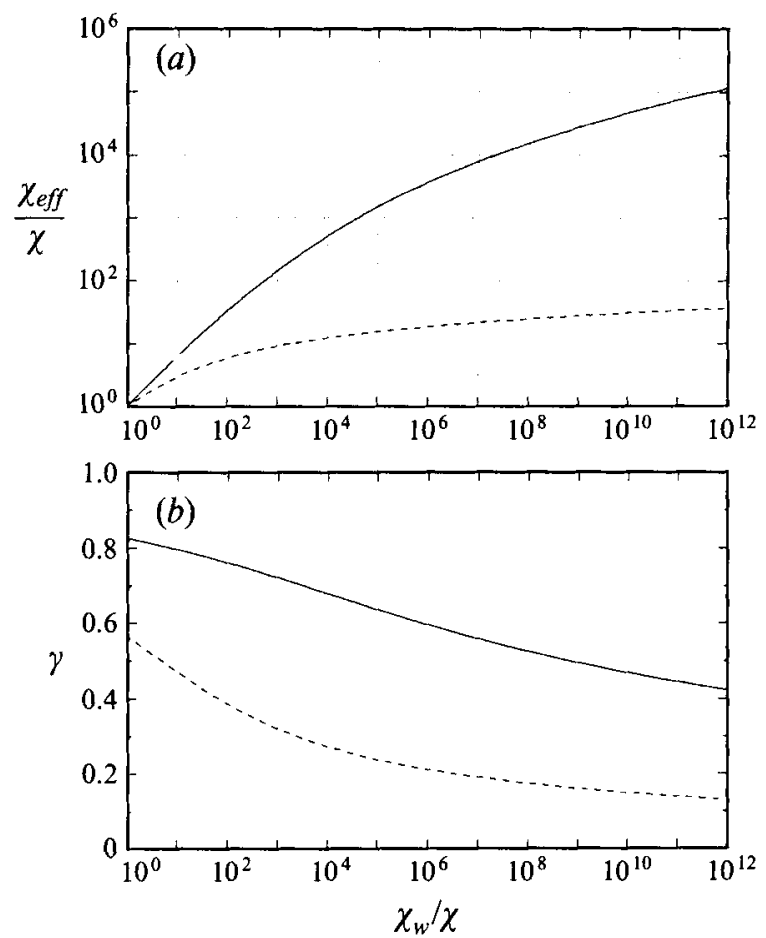

FigURE 11. Effective viscosity variation, $\chi_{e f f} / \tilde{\chi}$, plotted $(a)$ as a direct function of the viscosity variation $\chi_{w} / \tilde{\chi}$ across the compositional boundary layer; and $(b)$ as a power of $\chi_{w} / \tilde{\chi}$ (see equation (6.11)). The solid lines represent the effective viscosity given by matching the total upward compositional flux, while the dashed lines are obtained by matching the maximum upward velocity within the compositional boundary layer.

unimportant, compared with the effect of temperature, and the upward compositional flux is determined by the lower viscosity at the outer edge of the inner compositional boundary layer. However, when $\gamma$ is close to unity, the larger viscosity $\chi_{w}$ dominates.

In figure 11 , we plot $\chi_{\text {eff }} / \tilde{\chi}$ and the exponent $\gamma$ as functions of $\chi_{w} / \tilde{\chi}$. Figure 11 highlights the difference between our derived effective viscosity (solid lines) and that chosen by Nilson et al. (1985), who based their effective viscosity upon the maximum upward velocity within the compositional boundary layer (dotted line) and deduced that $\gamma$ may be set equal to the constant value $\frac{1}{5}$. From figure 11(b) we see that $\gamma=\frac{1}{5}$ indeed describes the maximum upward velocity for a viscosity variation of the order of $10^{6}$ across the compositional boundary layer, but that it is weighted too much towards the lower viscosity $\tilde{\chi}$ to provide an appropriate description of the total upward flux of composition. For viscosity variations up to about $10^{9}$ across the compositional boundary layer, we find instead that the effective viscosity should be weighted towards the larger viscosity $\left(\gamma>\frac{1}{2}\right)$, rather than the lower viscosity. As a result, the transport of fractionated fluid in a magma chamber is likely to be substantially diminished by the increase in viscosity associated with increased silica content and crystallinity close to the walls of a solidifying magma chamber, as suggested by Spera et al. (1982). For example, the expected box-filling rate predicted by Nilson et al. (1985) for a viscosity increase of $10^{6}$ across the compositional boundary layer should be reduced by an order of magnitude. 


$\begin{array}{ccccc}\text { Run } & \begin{array}{c}\text { Rayleigh } \\ \text { number }\end{array} & \begin{array}{c}\text { Thermal } \\ \text { variation }\end{array} & \begin{array}{c}\text { Compositional } \\ \text { variation }\end{array} & \begin{array}{c}\text { Effective } \\ \text { Rayleigh number }\end{array} \\ & R a_{C} & \frac{\tilde{\mu}}{\mu_{0}} & \frac{\mu_{w}}{\tilde{\mu}} & \frac{\mu_{0}}{\tilde{\mu}}\left(\frac{\tilde{\mu}}{\mu_{w}}\right)^{0.6} R a_{C} \\ 1 & 10^{6} & 33.3 & 3 & 1.55 \times 10^{4} \\ 2 & 10^{6} & 10 & 10 & 2.51 \times 10^{4} \\ 3 & 10^{6} & 1 & 100 & 6.31 \times 10^{4} \\ 4 & 10^{6} & 1 & 1 & 10^{6} \\ 5 & 10^{6} & 15.85 & 1 & 6.31 \times 10^{4} \\ 6 & 6.31 \times 10^{4} & 1 & 1 & 6.31 \times 10^{4} \\ 7 & 10^{5} & 1 & 10 & 2.51 \times 10^{4} \\ 8 & 2.51 \times 10^{4} & 1 & 1 & 2.51 \times 10^{4}\end{array}$

TABLE 2. Summary of numerical calculations performed for fluids of variable viscosity, with Rayleigh numbers based on the initial viscosity, $\mu_{0}$, of the fluid. The ratios $\tilde{\mu} / \mu_{0}$ and $\mu_{w} / \tilde{\mu}$ respectively describe the increase in viscosity across the thermal and compositional boundary layers, where $\mu_{w}$ is the fluid viscosity at the solidification front. For all calculations, $S t=5 ; \operatorname{Pr}=10 ; L e=50 ; A=0.9375$. The initial buoyancy ratio $\Gamma=R a_{T} / R a_{C}$ is equal to 0.1 in all cases. For a fluid with $S t=0.5$ and $L e=5 \times 10^{3}$, the same calculations apply to Rayleigh numbers a factor of $10^{10}$ greater. The effective Rayleigh number, $R a_{C} / \chi_{\text {eff }}$, is determined from the solutions to the inner compositional boundary layer outlined in $\$ 6.1$, and is the same both for Runs 3, 5 and 6 and for Runs 2, 7 and 8 .

\subsection{Numerical calculations}

To test the above analysis, a number of calculations were performed with a variety of Rayleigh numbers (based on $\mu_{0}$ ), and with differing viscosity differences across the thermal and compositional boundary layers (see table 2). The reader is referred to the Appendix for details of the numerical method. In comparing our results, we choose the effective viscosity by setting $\gamma=0.6$ in equation (6.11). This gives lower viscosities than the theoretical result (6.10) (see figure 11) for the range of viscosity variations studied here, but is still weighted in favour of the larger viscosity. We simply find that we obtain better agreement between calculations with this choice. The disparity is mainly due to the effect of the moving solid/liquid interface upon the boundary-layer structure. In the laboratory experiments and numerical calculations, the parameter $P \sim 0.12$ at least, while in magmatic situations, where $P$ can be much smaller, the above scaling analysis will work much better. With $\gamma=0.6$, the effective Rayleigh number, $R a_{C} / \chi_{e f f}$, is the same for each of the sets of Runs 3,5 and 6, and 2,7 and 8 .

Contours of fluid composition are plotted after a dimensionless calculation time $t=0.2$ for Runs 1-4 in figure 12, in which the solid region is shaded. The dimensionless effective viscosity, $\chi_{\text {eff }}$, decreases from Run 1 to Run 4 . Consequently, the box-filling rate is greatest for Run 4, and least for Run 1. In addition, the compositional boundary layer adjacent to the solid/liquid interface becomes noticeably thinner as the effective viscosity decreases, even though the total upward flow rate has increased. In Runs $1-3$, the viscosity increases from the interior towards the solid/liquid interface by a factor of 100 in all three cases, but the viscosity is essentially thermally determined for Run 1 (figure 12a), and is wholly compositionally determined for Run 3 (figure $12 c$ ). This demonstrates the greater importance of the thermal viscosity variation upon the box-filling rate.

In figure 13 , the vertical velocity profile is shown at the mid-height $y=0.5$ for the same four calculations. The strength of the upflow when there is no viscosity increase towards the solid/liquid interface (Run 4) is only about twice as great as 
(a)

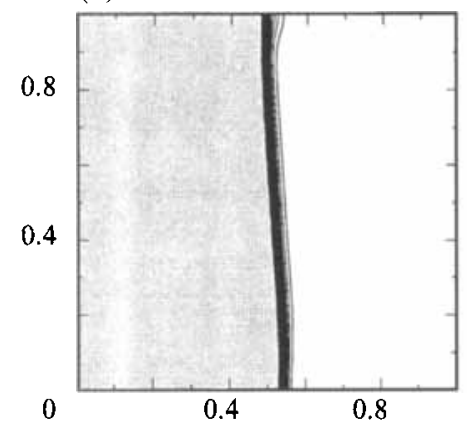

(c)

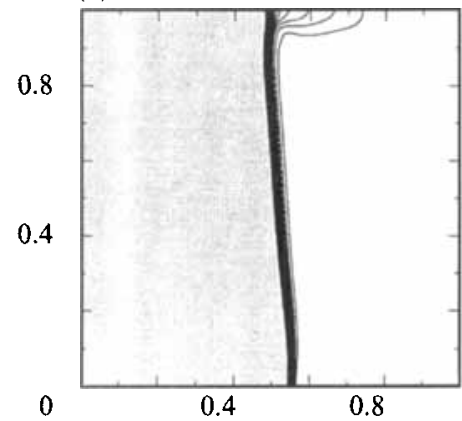

(b)

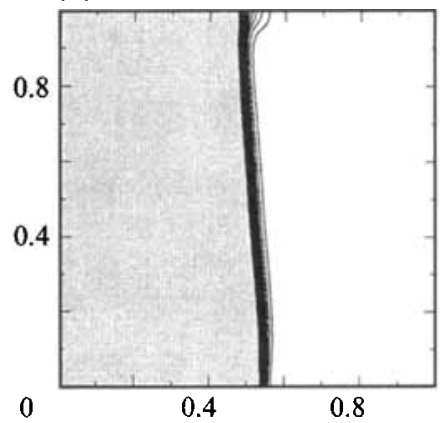

(d)

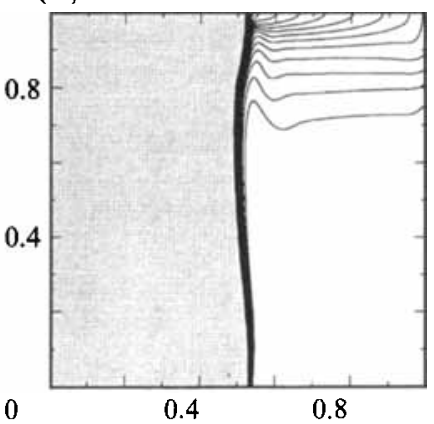

FIGURE 12. Contours of liquid composition for (a) Run 1 ; (b) Run 2; (c) Run 3 and $(d)$ Run 4 after a dimensionless time $t=0.2$; the contour interval is 0.05 . The effective viscosity $\chi_{\text {eff }}$ decreases from (a) to $(d)$. Parameter values are listed in table 2 . The initial far-field viscosity, $\mu_{0}$, is the same in all four cases, and fluid viscosity at the wall, $\mu_{w}$ is the same for Runs 1-3. In Run 4, viscosity is constant. Note that the compositional boundary layer at the solid/melt interface becomes thinner as $\chi_{\text {eff }}$ decreases.

when the viscosity increases by a factor of 100 owing to compositional differences alone (Run 3). However, the upflow is reduced by over an order of magnitude if the same viscosity difference is predominantly thermally determined (Run 1). This is because the compositional boundary layer is entirely contained within the highviscosity region.

The solid composition for Runs $1-4$ is presented in figure 14. For each calculation, two curves are plotted, the upper curve being the solid composition along the base of the chamber and the lower curve being the solid composition along the roof. The degree of vertical compositional stratification in the solid is represented by the difference between the upper and lower curves for each calculation. This stratification is a decreasing function of the effective viscosity $\chi_{\text {eff }}$, which is consistent with the variations in compositional boundary-layer thickness observed in figure 12 . The rapid decrease in solid composition close to the far boundary, $x=1$, for Run 4 reflects the relatively rapid box filling observed in that case, and if the calculation were continued, the solid composition would equal the eutectic composition at the far boundary.

Finally, in figure 15, we compare the solid composition at heights $y=0, y=0.5$ and $y=1$ for Runs 3,5 and 6 (figure 15a) and Runs 2, 7 and 8 (figure 15b). The agreement within each set is reasonably good, with the single exception of the lower curve for Run 3 (figure 15a), which describes the outflow region at the roof of the 


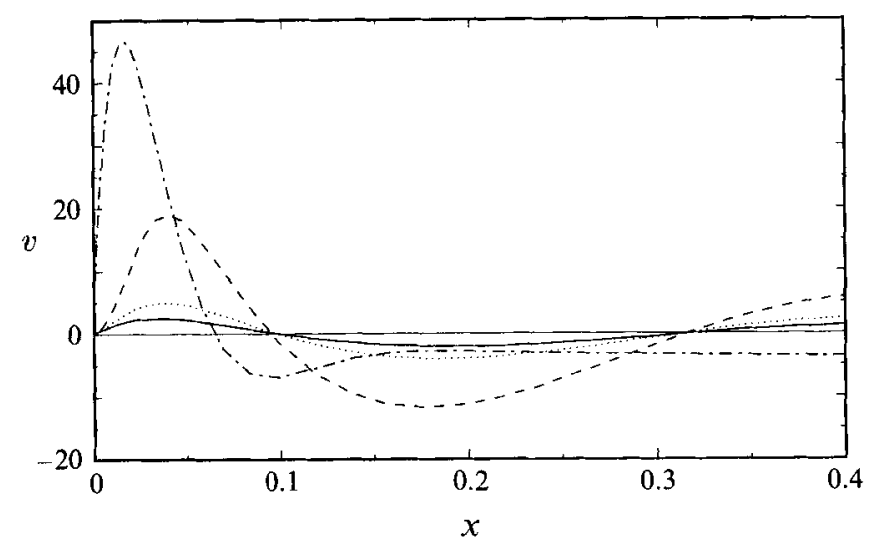

FIGURE 13. Upward velocity, $v$, plotted against distance $x$ from the solidification front at the mid-height $y=0.5$ after a dimensionless time $t=0.2$ for Run 1 (solid line), Run 2 (dotted line), Run 3 (dashed line) and Run 4 (dot-dashed line). As the effective viscosity $\chi_{\text {eff }}$ decreases from Run 1 to Run 4, the influence of the compositional buoyancy forcing over the thermal buoyancy forces increases, which further enhances the upward flux of light fractionated fluid.

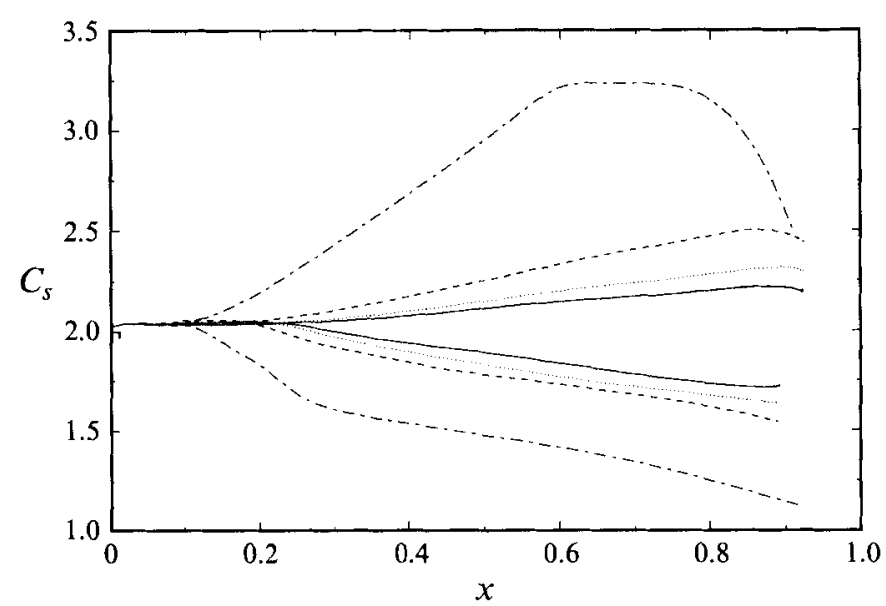

FIGURE 14. Dimensionless solid composition, $C_{s}$, plotted against distance $x$ from the cold wall for Run 1 (solid line), Run 2 (dotted line), Run 3 (dashed line) and Run 4 (dot-dashed line). For each run, two curves are plotted: the upper curve is the solid composition at the base, $y=0$, while the lower curve is the solid composition at the roof, $y=1$. The dimensionless eutectic composition is 1 and the initial melt composition is 2 . The effective viscosity $\chi_{\text {eff }}$ decreases from Run 1 to Run 4, and consequently the degree of vertical compositional stratification is greatest for Run 4 .

chamber. This is not surprising as the boundary-layer analysis in $\S 5$ is not appropriate to this region. The curves for Runs 5 and 6 (figure 15a) are almost indistinguishable, as are those for Runs 2 and 7 (figure $15 b$ ), which confirms our assumption that the region of upflow lies entirely within the thermal boundary layer for the regime studied here. Taken together, the two sets of runs offer good support for the scaling analysis in $\$ 6.1$, despite the slight disparity between the values for the effective viscosity given by equation (6.10), and the values given by substituting $\gamma=0.6$ into equation (6.11). 

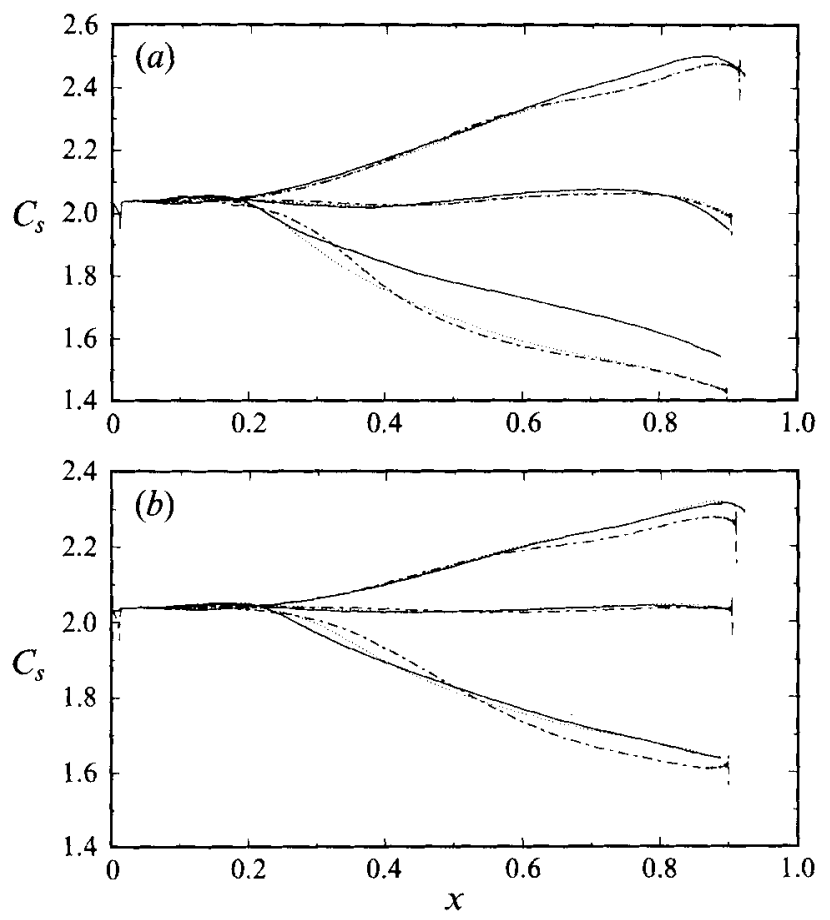

FIGURE 15. Dimensionless solid composition, $C_{s}$, plotted against distance $x$ from the cold wall for (a) Run 3 (solid line), Run 5 (dotted line) and Run 6 (dashed line); and (b) Run 2 (solid line), Run 7 (dotted line) and Run 8 (dot-dashed line). Within each set of runs, the effective Rayleigh number as given by equation (6.11) with $\gamma=0.6$ is the same (see table 2). Three curves are plotted for each run, corresponding to the roof $(y=1)$, mid-depth $(y=0.5)$ and the floor $(y=0)$.

\section{Discussion}

We have conducted a theoretical study of the compositional stratification in a solid grown from a liquid binary alloy by cooling at one sidewall. Our analysis is limited to fluids for which convection is laminar, and for which convection is compositionally dominated. By comparing the timescales for laminar box filling and for solidification, we are able to simulate directly experiments conducted at relatively high Rayleigh numbers, with fluids which have high Lewis numbers, by performing calculations at significantly lower Rayleigh and Lewis numbers. In the same manner, we are able to provide a model for solidification within a large magma chamber by extending our analysis to fluids whose viscosity is strongly dependent upon both temperature and composition. This represents a significant advance, despite the apparent simplicity of the scaling approach, because we are able to form a bridge between numerical calculations (which currently can only be performed over a limited parameter range), laboratory experiments and the expected conditions in a solidifying magma chamber.

We have tested our scaling theory by performing numerical calculations, including some direct comparisons with experiments reported in the literature. Despite some uncertainty concerning experimental conditions, agreement between our calculations and the experimental results is strong when the solid/liquid interface may reasonably be considered to be locally planar. Agreement is noticeably worse when the size of individual crystals becomes comparable to the characteristic thickness of the 
compositional boundary layer and turbulent plumes are released from the upper surfaces of the crystals (Leitch 1985). This demonstrates the fragility of theoretical and experimental results for laminar convection at a smooth vertical wall when they are applied to real systems.

One of our most important approximations has been that the advancing solid/liquid interface remains locally planar and that there is no morphological instability. It is not clear at present how appropriate this approximation is to crystallizing magmatic systems. An alternative end-member description is one in which binary alloys crystallize by forming two-phase regions, or mushes, in which interstitial fluid coexists in approximate thermodynamic equilibrium with a crystalline matrix. For example, many solidifying metallic alloys and aqueous salt solutions exhibit dendritic growth. Theoretical models for mushes have been proposed by Hills, Loper \& Roberts (1983), Huppert \& Worster (1985) and Worster (1986), and predictions made for the solid composition for the one-dimensional situation of a stagnant binary alloy cooled from below (Worster 1986), or a turbulently convecting fluid cooled from above (Kerr et al. 1990b). There have also been numerical calculations of convection in a porous mush driven by cooling from one side (Bennon \& Incropera 1987b; Beckermann \& Viskanta 1988; Voller, Brent \& Prakash 1989). Scaling analysis similar to that described in $\$ 4.2$ has been performed for porous flow by Lowell (1985), who suggests that the dimensionless box-filling timescale for a crystallizing mush layer should be proportional to $\bar{K}^{-1 / 2} R a_{C}^{-1 / 2} L e^{1 / 2}$. This would enable us to describe the compositional stratification in the solid in terms of the balance between solidification and box-filling timescales, but an accurate quantitative prediction for the solid composition is no longer possible. This is because solidification occurs over a finite interval when a mush is formed: between the time at which the mush front reaches a given position from the cold boundary and the time at which the eutectic front arrives at the same point. Hence the composition of the final solid product reflects some average of fluid conditions and is not an instantaneous snapshot of the flow structure.

Meanwhile, for the present model, direct application to magma chambers provides further challenges. Foremost among these is that magmas are not simple binary alloys, but are multi-component systems, which complicates the boundary-layer structure considerably. In particular, the exchange of water across the solidification front requires detailed consideration because of its potential to reduce significantly the viscosity of the magma close to the solid/liquid interface (Nilson et al. 1985; Spera, Oldenburg \& Yuen 1989). In addition, we have implicitly assumed a rather passive role for individual suspended crystals ahead of the solidification front. Typical calculations of magma viscosity below liquidus temperature include a contribution due to suspended crystals (e.g. Shaw 1972). Correct treatment of the region close to the solidification front therefore requires a sound description of the motion of these free crystals, and a determination of when such a partially solid region behaves rigidly.

In summary, it is imperative that more extensive use of scaling analysis be made to guide numerical modelling in the future. It may be that current models adequately represent the magmatic and metallurgical regimes because of the common practice of reducing Rayleigh numbers and Lewis numbers in simulations for reasons of numerical convenience. Such success, however, would be entirely fortuitous. Our analysis demonstrates that a wide range of styles of compositional stratification are possible, depending upon the value of the parameter $P$, and that care must be taken to ensure that the correct regime is being modelled. 
The authors have benefited from discussions with, and critical comments from, Paul Bruce, Mark Hallworth, Claude Jaupart, Ross Kerr, John Lister, Andrew Woods and Grae Worster. We are grateful to Arieh Iserles and Dan Moore for advice on numerical techniques, and to Alison Leitch for providing unpublished data from her original experiments. Financial support for R.A.J. was provided through a Benefactors' Studentship from St. John's College, Cambridge; the research of H.E.H. is partially supported by the Natural Environment Research Council.

\section{Appendix. Numerical methods}

The numerical methods outlined below are in general the same as those used by Thompson \& Szekely (1988) in a similar fluid-dynamical study. The reader is also referred to Peyret \& Taylor (1983) for details of the hydrodynamic method, and Crank (1984) for a description of the boundary-immobilization technique. Here, we shall describe our numerical method in only the barest terms, concentrating on those aspects, such as our treatment of variable vorticity, which are special to the present investigation.

First, the governing equations described in $\$ 3.1$ are non-dimensionalized using the scaling outlined in $\$ 3.2$. To eliminate the pressure term, the curl is taken of the dimensionless form of (3.1), leading to a streamfunction-vorticity formulation. The full advection-diffusion equations for the fluid temperature and composition differ from equations (3.11) and (3.12) only in the reinstatement of the partial derivative in $t$ on each left-hand side, and the second partial derivative in $y$ on each right-hand side, while the momentum equation (3.1) becomes the vorticity equation

$$
\frac{1}{\operatorname{Pr}}\left(\frac{\partial \omega}{\partial t}+\boldsymbol{u} \cdot \nabla \omega\right)=R a_{T} \frac{\partial T}{\partial x}-R a_{C} \frac{\partial C}{\partial x}+\nabla^{2}(\chi \omega)+2 \varepsilon_{3 j k} \frac{\partial u_{i}}{\partial x_{k}} \frac{\partial^{2} \chi}{\partial x_{i} \partial x_{j}},
$$

where

$$
\nabla^{2} \psi=-\omega
$$

Here, $\psi$ is the streamfunction and $\omega=\boldsymbol{k} \cdot \nabla \wedge \boldsymbol{u}$ is the sole non-zero component of vorticity. The boundary condition for the streamfunction is simply $\psi=0$, while the boundary conditions for the vorticity, $\omega$, are derived from the no-slip condition, $\boldsymbol{u}=0$. Following Peyret $\&$ Taylor (1983), we write

$$
\psi(x, \Delta y)=\psi(x, 0)+\Delta y \frac{\partial \psi}{\partial y}(x, 0)+\frac{1}{2}(\Delta y)^{2} \frac{\partial^{2} \psi}{\partial y^{2}}(x, 0)+\cdots,
$$

for the region close to the boundary $y=0$. The condition $u=0$ implies that $\psi(x, 0)=\psi_{y}(x, 0)=0$, and hence from (A 2), the boundary vorticity, $\omega(x, 0)$, may be estimated to leading order from the (assumed known) streamfunction at the first interior grid point by writing

$$
\omega(x, 0)=-\frac{2}{(\Delta y)^{2}} \psi(x, \Delta y) .
$$

In a similar manner, we may derive the boundary vorticity from the no-slip condition at the remaining fluid boundaries $y=1, x=X$ and $x=1$. Higher-order forms of (A 4) typically led to numerical instability.

Potentially, variable viscosity can cause difficulties when attempting to find numerical solutions. These difficulties arise from the possibility of source terms, such as $\omega \nabla^{2} \chi$, appearing on the right-hand side of the vorticity equation. Here, we have 
avoided such problems by writing the curl of the viscous dissipation as the sum of the final two terms in (A 1). In solving (A 1), the term $\nabla^{2}(\chi \omega)$ is not expanded, but is discretised as it is written. The final term in (A 1) still provides a source term in the vorticity, but it is simple to show that for the problem studied here it is much smaller in magnitude than the buoyancy terms outside a viscous boundary layer of thickness $\delta_{v} \ll L$, and that it is smaller than $\nabla^{2}(\chi \omega)$ by a factor of order $\left(\delta_{v} / L\right)^{2}$ within the viscous boundary layer. Hence the final term may be neglected in the numerical calculations. The small magnitude of the neglected term has also been confirmed $a$ posteriori.

The dimensionless transport equations can be transformed further by mapping each of the irregularly shaped solid and liquid regions onto the domain $[0,1] \times$ $[0,1]$, a technique known as boundary immobilization (Crank 1984). In return for simplifying the calculation domain, additional terms enter into the transport equations. These terms can be divided into two classes: interface curvature terms, which are proportional to $X_{y}$ and $X_{y y}$, and pseudo-advective terms, which are proportional to $X_{t}$. Many workers (e.g. Ramachandran et al. 1981; Ho \& Viskanta 1984; Thompson \& Szekely 1988) have chosen to neglect most of the additional terms introduced by this transformation, including the pseudo-advective terms, as part of a pseudostationary approximation. This approximation significantly enhances convergence of the numerical scheme. Tests with the current model confirm that, except at very early times, these terms are indeed negligible when only the position of the solidification front is required. However, neglect of the pseudo-advective terms leads to significant numerical errors in the compositional flux at the solid/liquid interface. Hence, we retain the pseudo-advective terms in our present study, but neglect the interface curvature terms.

The transformed parabolic advection-diffusion equations for the solid temperature, and for the fluid vorticity, temperature and composition, are solved using the Alternating Direction Implicit (ADI) method introduced by Peaceman \& Rachford (1955), with upwinding for the advective terms. For the potentially expensive inversion $\nabla^{2} \psi=-\omega$, we employ a multigrid method, as described by Sonneveld, Wesseling $\&$ de Zeeuw (1985). The equations were solved on an irregular mesh with $21 \times 41$ grid points in the solid domain, and $65 \times 41$ grid points in the fluid domain. The dimensionless grid spacing in the fluid close to the solidification front is approximately $3.3 \times 10^{-5}$, well within the expected thickness of the compositional boundary layer at mid-height. For constant-coefficient parabolic equations, the ADI method is unconditionally stable (Peyret \& Taylor 1983), and the choice of timestep is determined by temporal accuracy in determining $\dot{X}$. Our initial timestep is $\Delta t=10^{-5}$, which is steadily increased as the calculation progresses, whenever $\dot{X} \Delta t$ becomes smaller than $10^{-4}$, to a maximum value $\Delta t=2 \times 10^{-3}$.

The couplings inherent in the governing equations and boundary conditions are treated by performing a global iterative procedure at each timestep. Each equation is solved in turn as if all other variables are already known at the new timestep, including the solidification rate $\dot{X}$, which is deduced from the dimensionless form of the interfacial thermal boundary condition (3.9). This procedure is then repeated until convergence at the new timestep is achieved. The global iteration also enables us to perform a third-order correction to the upwinded advective terms while solving the parabolic equations. The use of higher-order upwinding means that the total composition is typically conserved through an entire crystallization calculation to within $0.1 \%$. Simple first-order upwinding only allows mass to be conserved to within $5 \%$. 
The calculations are started at the dimensionless time $t=10^{-4}$ from the similarity solution for the solidification of a binary alloy in the absence of convection, as described by Worster (1986), thereby avoiding the singularity in the solidification rate as $t \rightarrow 0$. A typical calculation took approximately $10 \mathrm{cpu}$ hours on a DECStation 5000 .

\section{REFERENCES}

BECKERMANN, C. \& VISKANTA, R. 1988 Double-diffusive convection during dendritic solidification of a binary mixture. PhysicoChem. Hydrodyn. 10, 195-213.

BENNON, W. D. \& INCROPERA, F. P. 1987a A continuum model for momentum, heat and species transport in binary solid-liquid phase change systems - I. Model formulation. Intl J. Heat Mass Transfer 30, 2161-2170.

BENNON, W. D. \& INCROPERA, F. P. $1987 b$ A continuum model for momentum, heat and species transport in binary solid-liquid phase change systems - II. Application to solidification in a rectangular cavity. Intl J. Heat Mass Transfer 30, 2171-2187.

Chen, C.-F. \& Turner, J. S. 1980 Crystallization in a double-diffusive system. J. Geophys. Res. 85, 2573-2593.

Crank, J. 1984 Free and Moving Boundary Problems. Oxford University Press.

EllioT, R. 1977 Eutectic solidification. Intl Metals Revs. 219, 161-186.

Gebhart, B. \& Pera, L. 1971 The nature of vertical natural convection resulting from combined buoyancy effects of thermal and mass diffusion. Intl J. Heat Mass Transfer 14, 2025-2050.

HeBDitch, D. J. 1975 Contribution concerning the solidification problem. In Moving Boundary Problems in Heat Flow and Diffusion (ed. J. R. Ockendon \& W. R. Hodgkins). Oxford University Press.

HILDRETH, W. 1981 Gradients in silicic magma chambers: implications for lithospheric magmatism. J. Geophys. Res. 86, 10153-10192.

Hills, R. N., Loper, D. E. \& RoberTs, P. H. 1983 A thermodynamically consistent model of a mushy zone. Q. J. Appl. Maths 36, 505-539.

Ho, C.-J. \& ViskANTA, R. 1984 Heat transfer during melting from an isothermal vertical wall. Trans. ASME C: J. Heat Transfer 106, 12-19.

Huppert, H. E. 1990 The fluid mechanics of solidification. J. Fluid Mech. 212, 209-240.

Huppert, H. E., Sparks, R. S. J., Wilson, J. R., Hallworth, M. A. \& Leitch, A. M. 1987 Laboratory experiments with aqueous solutions modelling magma chamber processes II. Cooling and crystallization along inclined planes. In Origins of Igneous Layering (ed. I. Parsons), pp. 539-568. Reidel.

HUPPERT, H. E. \& WORSter, M. G. 1985 Dynamical solidification of a binary melt. Nature 314, 703-707.

JARVIS, R. A. 1991 Crystallization and melting in geological fluid mechanics. PhD thesis, University of Cambridge.

Kerr, R. C., Woods, A. W., Worster, M. G. \& Huppert, H. E. 1990 a Solidification of an alloy cooled from above. Part 2. Non-equilibrium interfacial kinetics. J. Fluid Mech. 217, 331-348.

Kerr, R. C., Woods, A. W., Worster, M. G. \& Huppert, H. E. $1990 b$ Solidification of an alloy cooled from above. Part 3. Compositional stratification within the solid. J. Fluid Mech. 218, 337-354.

Kuiken, H. K. 1968 An asymptotic solution for large Prandtl number free convection. J. Engng Maths 2, 355-371.

LANGER, J. S. 1980 Instabilities and pattern formation in crystal growth. Rev. Mod. Phys. 52, 1-28.

LerTCH, A. M. 1985 Laboratory models of magma chambers. PhD thesis, Australian National University.

LerTCH, A. M. 1987 Various aqueous solutions crystallizing from the side. In Structure and Dynamics of Partially Solidified Systems (ed. D. E. Loper), pp. 37-57. Nijhoff.

LerTCH, A. M. 1990 Free convection in laboratory models. Earth-Sci. Revs. 29, 369-383.

Lowell, R. P. 1985 Double-diffusive convection in partially molten silicate systems: its role during magma production and in magma chambers. J. Volcanol. Geotherm. Res. 26, 1-24. 
MCBIRNEY, A. R. \& Noyes, R. M. 1979 Crystallization and layering of the Skaergaard Intrusion. J. Petrol. 20, 487-554.

Mulins, W. W. \& Sekerka, R. F. 1964 Stability of a planar interface during solidification of a dilute binary alloy. J. Appl. Phys. 35, 444-451.

NiLson, R. H. 1985 Countercurrent convection in a double-diffusive boundary layer. J. Fluid Mech. 160, 181-210.

Nilson, R. H. \& BAER, M. R. 1982 Double diffusive counterbuoyant boundary layer in laminar natural convection. Intl J. Heat Mass Transfer 25, 285-287.

Nilson, R. H., McBirney, A. R. \& Baker, B. H. 1985 Liquid fractionation. Part II: Fluid dynamics and quantitative implications for magmatic systems. J. Volcanol. Geotherm. Res. 24, 25-54.

OstraCH, S. 1953 An analysis of laminar free convection and heat transfer about a vertical flat plate. NACA Rep. 1111.

Peaceman, D. W. \& RaChFord, H. H. 1955 The numerical solution of parabolic and elliptic differential equations. J. Soc. Indust. Appl. Maths 3, 28-41.

Peyret, R. \& Taylor, T. D. 1983 Computational Methods for Fluid Flow. Springer.

RAmachandran, N., Gupta, J. P. \& JaluRia, Y. 1981 Two-dimensional solidification with natural convection in the melt and convective and radiative boundary conditions. Num. Heat Transfer 4, $469-484$.

SHAW, H. R. 1972 Viscosities of magmatic silicate liquids: an empirical method of prediction. $A m$. J. Sci. 272, 870-893.

Sonneveld, P., Wesseling, P. \& Zeeuw, P. M. DE 1985 Multigrid and conjugate gradient methods as convergence acceleration techniques. In Multigrid Methods for Integral and Differential Equations (ed. D. J. Paddon \& H. Holstein), pp. 117-167. Oxford University Press.

Spera, F. J., Oldenburg, C. M. \& Yuen, D. A. 1989 Magma zonation: effects of chemical buoyancy and diffusion. Geophys. Res. Lett. 16, 1387-1390.

Spera, F. J., YUEn, D. A. \& Kirschvink, S. J. 1982 Thermal boundary layer convection in silicic magma chambers: effect of temperature-dependent rheology and implications for thermogravitational chemical fractionation. J. Geophys. Res. 87, 8755-8767.

Thompson, M. E. \& SZeKely, J. 1987 Double-diffusive convection during solidification at a vertical wall. In Structure and Dynamics of Partially Solidified Systems (ed. D. E. Loper), pp. 59-77. Nijhoff.

Thompson, M. E. \& Szekely, J. 1988 Mathematical and physical modelling of double-diffusive convection of aqueous solutions crystallizing at a vertical wall. J. Fluid Mech. 187, 409-434.

TuRnER, J. S. \& Gustafson, L. B. 1981 Fluid motions and compositional gradients produced by crystallization or melting at vertical boundaries. J. Volcanol. Geotherm. Res. 11, 93-125.

Voller, V. R., Brent, A. D. \& Prakash, C. 1989 The modeling of heat, mass and solute transport in solidification systems. Intl J. Heat Mass Transfer 32, 1719-1731.

WEAST, Q. C. (Ed.) 1971 Handbook of Chemistry and Physics. Cleveland Rubber Company.

Woods, A. W. \& HuPPERT, H. E. 1989 The growth of compositionally stratified solid above a horizontal boundary. J. Fluid Mech. 199, 29-54.

Worster, M. G. 1986 Solidification of an alloy from a cooled boundary. J. Fluid Mech. 167, 481-501.

Worster, M. G. 1992 The dynamics of mushy layers. In Interactive Dynamics of Convection and Solidification (ed. S. H. Davis, H. E. Huppert, U. Müller \& M. G. Worster). NATO ASI E219, pp. 113-138. Kluwer.

Worster, M. G. \& Leitch, A. M. 1985 Laminar free convection in confined regions. J. Fluid Mech. 156, 301-319. 